\title{
Hierarchical Pigeon-Inspired Optimization- based MPPT Method for Photovoltaic Systems under Complex Partial Shading Conditions
}

\author{
Zhuoli Zhao, Member, IEEE, Mingyu Zhang, Zehan Zhang, Yuewu Wang, Runting Cheng, Juntao \\ Guo, Ping Yang, Chun Sing Lai, Senior Member, IEEE, Peng Li, Loi Lei Lai, Life Fellow, IEEE
}

\begin{abstract}
This paper proposes a novel maximum power point tracking (MPPT) method based on the variant of the pigeon-inspired optimization (PIO) algorithm for photovoltaic (PV) systems under partial shading conditions (PSCs). The proposed method integrates the hierarchical network behavior of pigeon flock and revises the map and compass operator of the original PIO algorithm to improve optimization efficiency. In addition, the landmark operator is used to perform a small-scale search to achieve fast tracking. Based on the combination of these mechanisms and dual-mode dynamic tracking scheme, the proposed hierarchical pigeon-inspired optimization (HPIO) MPPT method has a powerful search ability to deal with PSCs. To verify the superiority of the proposed HPIO MPPT method, it is compared with other existing advanced MPPT methods in simulation and experiments. Compared with traditional MPPT techniques based on artificial intelligence, the proposed HPIO MPPT method has a higher success rate in tracking GMPP and excellent tracking speed under PSCs. And the HPIO method also shows excellent performance under complex PSC with multiple clusters and load-variation conditions.
\end{abstract}

This work was supported by the National Natural Science Foundation of China 51907031; Guangdong Basic and Applied Basic Research Foundation (Guangdong-Guangxi Joint Foundation) 2021A1515410009; Brunel Research Initiative and Enterprise Fund (BRIEF). (Corresponding authors: Loi Lei Lai; Chun Sing Lai)

Z. Zhao, M. Zhang, Z. Zhang, R. Cheng, J. Guo, and L. L. Lai are with the Department of Electrical Engineering, School of Automation, Guangdong University of Technology, Guangzhou, 510006, China (e-mail: zhuoli.zhao@gdut.edu.cn; mingyu@mail2.gdut.edu.cn; gdut3119001299@mail2.gdut.edu.cn; chengrunting@mail2.gdut.edu. cn;2111904003@mail2.gdut.edu.cn; I.I.lai@ieee.org).

Y. Wang is with the School of Electric and Information Engineering, Guangxi University of Science and Technology, Liuzhou 545006, China (e-mail: wangyuewu@gxust.edu.cn).

P. Yang is with the Guangdong Key Laboratory of Clean Energy Technology, South China University of Technology, Guangzhou 510640 China (e-mail: eppyang@scut.edu.cn).

C. S. Lai is with Brunel Interdisciplinary Power Systems Research Centre, Department of Electronic \& Electrical Engineering, Brunel University London, London, UB8 3PH, UK and also with the Department of Electrical Engineering, School of Automation, Guangdong University of Technology, Guangzhou, 510006, China (e-mail: chunsing.lai@brunel.ac.uk).

P. Li is with the Digital Grid Research Institute of China Southern Power Grid, Guangzhou 510663, China (e-mail: lipeng@csg.cn).
Index Terms-Photovoltaic (PV) systems, maximum power point tracking, partial shading conditions, hierarchical pigeon-inspired optimization

\section{INTRODUCTION}

T $\mathrm{N}$ recent years, photovoltaic $(\mathrm{PV})$ power generation has attracted more and more attention because of the advantages of low operating cost and environmental friendliness. Since the maximum power point (MPP) of PV system changes with environmental conditions, MPP tracking (MPPT) has become a challenging task. PV array is composed of diodes and panels connected in series and parallel. When uneven light is irradiated on the PV array, partial shading conditions (PSCs) are formed. Thus, the diode causes the power-voltage (P-V) curve to have multiple peaks, and the realization of MPPT faces significantly increased difficulty [1].

Traditional MPPT algorithms, including perturbation and observation (P\&O) algorithm [2], incremental conductance (IC) algorithm [3] and hill-climbing (HC) algorithm [4], were improved that adapts to rapid changes in irradiation. Conventional MPPT algorithms developed in the past are only suitable for PV systems with a single peak under uniform sunlight conditions, and they cannot track GMPP under PSCs effectively. In recent years, the application of soft computing methods to solve the MPPT problem under PSCs has become a hot topic. In [5]-[6], the use of artificial neural networks (ANN) and fuzzy logic control (FLC) to successfully track GMPP under PSCs were reported. However, these methods require extensive experience and involve complex calculations, which are not conducive to practical applications. In [7], a novel maximum power point scanning (MPPS) technique was developed, which can be integrated into the online or offline tester. [8] adopted an intelligent mechanism to systematically schedule the MPPT process, and used a novel skipping scheme to further minimize the search region.

Bio-inspired algorithms are a new and important branch in the field of artificial intelligence (AI) which can well solve nonlinear and stochastic optimization problems and effectively improve the reliability of practical applications. Bio-inspired algorithms have two main categories: evolutionary algorithms and algorithms based on swarm intelligence. Typical evolutionary algorithms combined with MPPT applications are genetic algorithm (GA) [9] and differential evolution (DE) [10]. Reference [11] used GA to obtain the best member functions and control rules for FLC to improve the MPPT performance. 


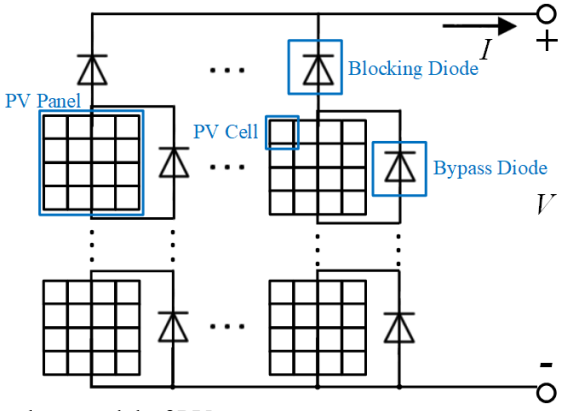

Fig. 1. Equivalent model of PV array.

Reference [12] further improved the MPPT method based on DE, which can search for GMPP in a larger working area, thereby improving the ability to track GMPP.

In recent years, many swarm intelligence (SI) algorithms have been developed and applied to achieve MPPT for PV systems. A hybrid MPPT method combined the adaptive P\&O and particle swarm optimization (PSO), which is proposed in [13], proved that it has a real global tracking effect with short tracking time. [14] developed a novel method called adaptive radial movement optimization (ARMO), which can maximize convergence speed and output fluctuation during tracking. In [15], a hybrid MPPT method combining adaptive neuro-fuzzy in reference system and particle swarm optimization was applied, which can track the MPP rapidly with zero oscillation. Reference [16] developed a novel MPPT algorithm based on Flying Squirrel Search Optimization (FSSO), which showed a higher convergence speed and performed better than P\&O algorithm and PSO algorithm. Reference [17] proposed a hybrid tracking technology, which can switch between traditional P\&O and Flower Pollination Algorithm (FPA) by accurately detecting shading conditions. Reference [18] used improved fusion firefly algorithm (FFA) to track the GMPP under PSCs, which integrated the neighborhood attraction firefly algorithm (NaFA) and simplified firefly algorithm (SFA), and achieved a high efficiency to track the GMPP. Reference [19] presented a logarithmic PSO method, which reduced the power oscillation during the MPPT process and accelerated the convergence. In [20], a modified butterfly optimization algorithm (MBOA) was developed to solve the MPPT problem under PSCs, which distinguished different partial shadow conditions with fast convergence speed.

The above studies have shown that various bio-inspired AI-based algorithms have better performance than traditional MPPT algorithms such as P\&O, IC and HC, with higher tracking accuracy and faster tracking response. However, the selection of parameters and initial values has a great impact on the tracking performance of bio-inspired AI-based algorithms [21]. Inappropriate parameters and initial values may lead to local MPP (LMPP) or too long tracking time. Therefore, when designing parameters and initial values, it is necessary to find a balance between the three indicators of tracking success rate, tracking efficiency and tracking speed.

Inspired by the behavior of homing pigeons in navigation, Duan et al. [22] proposed pigeon-inspired optimization (PIO) in 2014, which is a new SI algorithm. Pigeons can perceive the earth's magnetic field and use the sun as a compass to generate a map in their brains to convert the direction, which is relative to the sun, into the actual flight direction. At the same time,

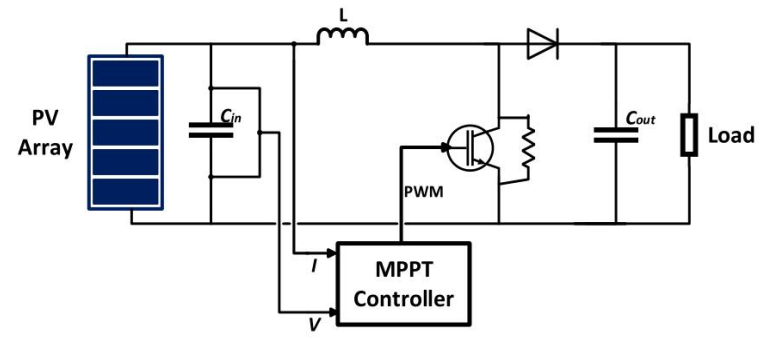

Fig. 2. The block diagram of the studied PV system. TABLE I

IRRADIANCE VALUES OF FOUR CASES

\begin{tabular}{ccccccc}
\hline \hline \multirow{2}{*}{ Cases } & \multicolumn{7}{c}{ Irradiance Values of PV panels $\left(\mathrm{W} / \mathrm{m}^{2}\right)$} \\
& Panel 1 & Panel 2 & Panel 3 & Panel 4 & Panel 5 & $P_{\max }$ \\
\hline Case 1 & 1000 & 1000 & 1000 & 1000 & 1000 & $498.4 \mathrm{~W}$ \\
Case 2 & 1000 & 875 & 750 & 625 & 500 & $294.4 \mathrm{~W}$ \\
Case 3 & 1000 & 875 & 750 & 625 & 375 & $281.9 \mathrm{~W}$ \\
Case 4 & 1000 & 875 & 375 & 250 & 125 & $172.9 \mathrm{~W}$ \\
\hline \hline
\end{tabular}
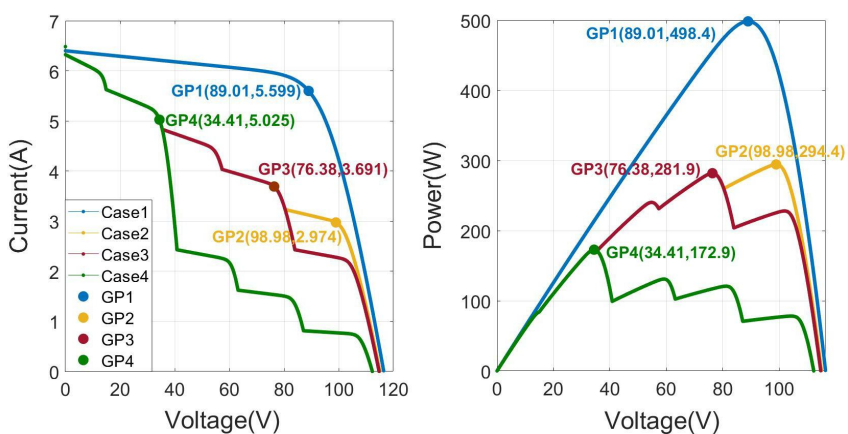

Fig. 3. I-V and $\mathrm{P}-\mathrm{V}$ characteristic curves of $\mathrm{PV}$ arrays under four cases.

pigeons have the memory to distinguish familiar landmarks, so that they can reach their destination fast and accurately. PIO is built on the above-mentioned biological basis. In applications of unmanned aerial vehicle, PIO shows excellent performance with good convergence and high efficiency [23]. However, similar to other bio-inspired algorithms, PIO still has the general problem of premature convergence [24].

Motivated by the aforementioned research gap, this paper improves the PIO algorithm, and proposes a hierarchical network in the flock of pigeons to revise the map and compass operator, so as to improve the optimization efficiency. Moreover, the landmark operator also ensures fast convergence to achieve fast tracking speed. Therefore, the proposed hierarchical pigeon-inspired optimization (HPIO) method can quickly and efficiently track GMPP under various PSCs. On the basis of above improvement, a tracking technique that switches between the HPIO and P\&O algorithm in two modes is proposed. Simulations are carried out under 56 PSCs, and experiments are conducted for several cases. By comparing the other three existing advanced MPPT methods, the superiority of the proposed MPPT method is verified. The proposed MPPT method, which can switch between two MPPT modes, can be directly applied in actual operation and has good practicability. The contributions of this paper are listed as follows:

1) Based on the research of hierarchical group dynamics in pigeon flock, a hierarchical network is constructed to revise map and compass operator of the original PIO algorithm, thus enhancing the global search ability and improving the tracking efficiency to track GMPP under PSCs. 
2) A dual-mode dynamic tracking technique that switches between two MPPT modes is proposed. "Intelligent Mode" quickly determines the approximate position of GMPP, while "Disturbance Mode" maintains efficient dynamic tracking.

3) The proposed HPIO method is compared with other MPPT methods in specific cases and dynamic tracking tests with verification results. Tests under complex PSC with multiple clusters and tests under load-variation are also conducted to verify the effectiveness of the proposed method.

\section{II.MODELING OF PV ARRAY AND CHARACTERISTICS UNDER PSCS}

Assuming that the number of series and parallel are $N_{s}$ and $N_{p}$, respectively, the I-V characteristics of the PV panel are [25]:

$$
I=N_{p} I_{p v}-N_{p} I_{0}\left[\exp \left(\frac{q V+q R_{s} I N_{s} / N_{p}}{N_{s} k_{s} T a}-1\right)\right]-\frac{V N_{p} / N_{s}+R_{s} I}{R_{p}}
$$

PV array is formed by connecting multiple PV panels in parallel or series, as shown in Fig. 1. The PV panel shaded under PSCs will cause the hot spot effect. To prevent damage to the PV panel due to overheating, it is necessary to connect a bypass diode to the PV panel in parallel. However, due to the conduction of the diode, the output characteristics of the PV array under PSCs will have multiple extreme points, and this phenomenon is obvious in a PV array composed of multiple PV panels in series.

Fig. 2 shows the studied PV system, and the PV array consists of five modules connected in series. Table I shows the different irradiance combinations for the four GMPP locations. Fig. 3 shows the I-V and P-V characteristic curves of the four cases. In Case 1, the PV array receives the same radiance. Among the other three PSCs, the five PV panels of the PV array receive five different irradiances, and there are five MPPs on the P-V characteristic curve, but the location of GMPP is different. In Case 2, GMPP is located at GP2 near 85\% Voc. In Case 3, GMPP is located at GP3 near $30 \%$ Voc. In Case 4, GMPP is located at GP4 near $65 \%$ Voc.

\section{The Proposed Method}

\section{A. Principle of PIO Algorithm}

Homing pigeons have played a significant role in transmitting information in human history. Studies have found that the excellent homing ability of pigeons depends on the ability to use the geomagnetic field and the sun to determine the direction, which play the role of "map and compass" [22]. As the pigeon approaches the destination, the navigational role of the "map and compass" gradually weakens, and the navigation tools are eventually replaced with landmarks 26]. Inspired by the behaviors in homing pigeon navigation, Duan et al. proposed pigeon-inspired optimization (PIO) [22]. This novel bio-inspired intelligence algorithm includes two operators: map and compass operator, landmark operator. The former emphasizes the influence of the sun and the geomagnetic field on navigation, while the latter emphasizes the influence of landmarks. The process of the PIO algorithm can be divided into two parts:

\section{1) Map and Compass Operator}

In multi-dimensional search space, the position and speed of the pigeons are initialized and will be updated in each iteration.

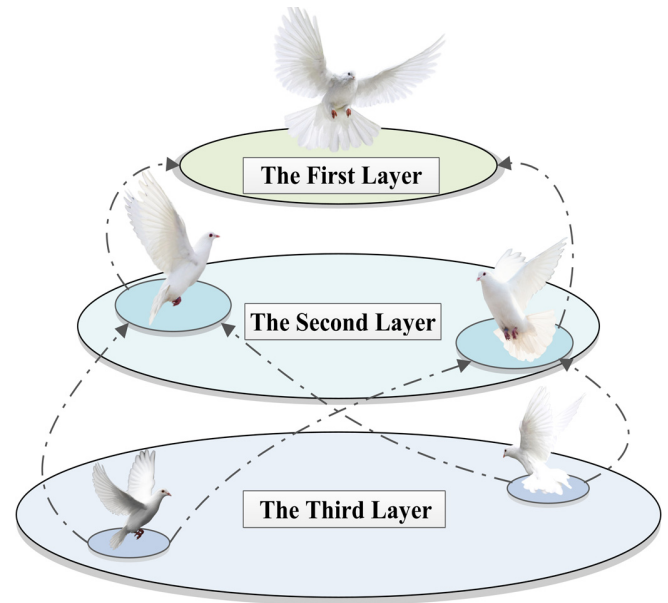

Fig. 4. The conceptual diagram of the hierarchical network of pigeon flocks. Its position and speed are denoted as $x_{i}$ and $v_{i}(i=1,2, \cdots, N)$. During each iteration, the pigeon flies toward the global optimal position and maintains a certain speed inertia, which makes the PIO algorithm have an excellent global search capability. Each pigeon updates its position $x_{i}$ and speed $v_{i}$ by:

$$
\begin{gathered}
v_{i}^{k}=v_{i}^{k-1} \cdot e^{-R \cdot k}+\operatorname{rand} \cdot\left(x_{\text {gbest }}-x_{i}^{k-1}\right) \\
x_{i}^{k}=x_{i}^{k-1}+v_{i}^{k}
\end{gathered}
$$

where $R$ is the map and compass factor, the value range is set to $0 \sim 1$; rand is a random number with a value range of $0 \sim 1 ; k$ is the current iteration number; $x_{\text {gbest }}$ is the global optimal position obtained after $k$ - 1 iteration loops.

\section{2) Landmark Operator}

When approaching the destination, the pigeons will rely on nearby landmarks to optimize their position. The pigeons which are familiar with the landmark will fly directly to the destination, while other pigeons that are not familiar with the landmark will follow the former. According to the principle of the landmark operator, the number of pigeons will be reduced by half after each iteration. $x_{\text {center }}$ is the center position of the remaining pigeons and will be used as a landmark. Therefore, $x_{i}$ is updated according to:

$$
\begin{gathered}
N^{k}=\frac{N^{k-1}}{2} \\
x_{\text {center }}^{k}=\frac{\sum_{i=1}^{N^{k}} x_{i}^{k-1} \cdot F_{i}^{k-1}}{N^{k} \cdot \sum_{i=1}^{N^{k}} F_{i}^{k-1}} \\
F_{i}^{k-1}=\left\{\begin{array}{l}
x_{i}^{k}=x_{i}^{k-1}+\operatorname{rand} \cdot\left(x_{\text {center }}^{k}-x_{i}^{k-1}\right) \\
\frac{1}{\text { Fitness }\left(x_{i}^{k-1}\right)+\varepsilon}, \text { for minimization } \\
\text { Fitness }\left(x_{i}^{k-1}\right), \quad \text { for maximization }
\end{array}\right.
\end{gathered}
$$

where $\varepsilon$ is a constant close to zero to ensure the validity of the equation. When the landmark operator stops, the PIO ends the iteration and obtains the historical global optimal position $x_{p}$.

\section{B. The proposed Hierarchical PIO (HPIO)}

Analyzing the map and the compass operator, it can be found that the conventional PIO algorithm is easy to fall into the local extreme value. In the early stage of the PIO algorithm, it is hoped to have a strong global search capability, so as to keep 


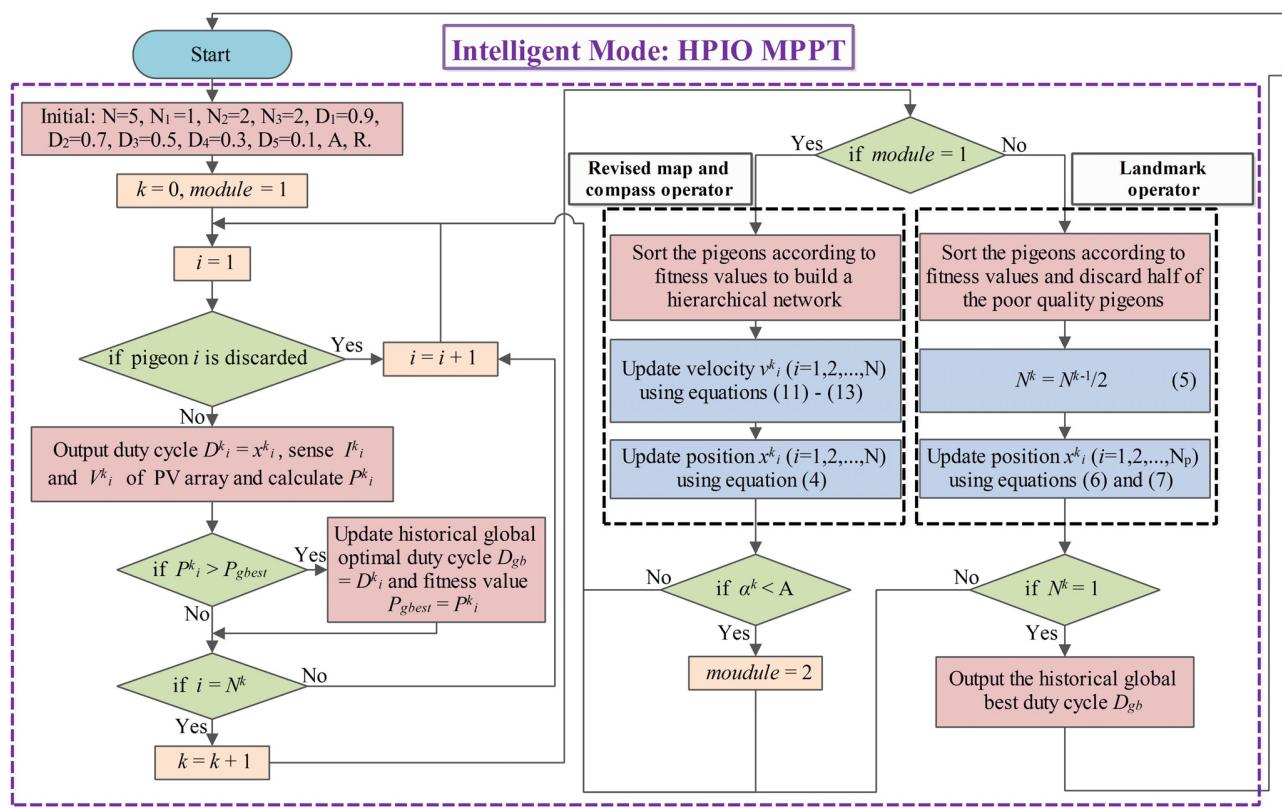

Disturbance Mode: P\&O MPPT

Fig. 5. Flowchart of the proposed MPPT method combined with HPIO and P\&O MPPT algorithms.
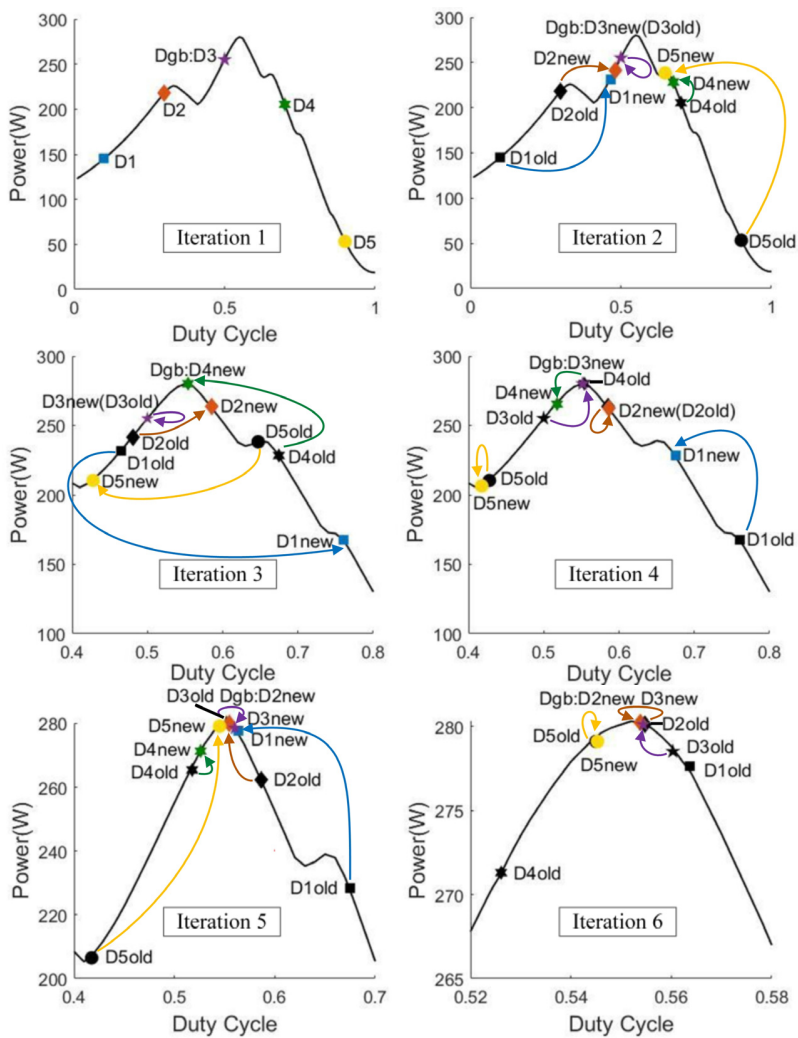

Fig. 6. Position changes of the pigeon flock during a single tracking process.

away from converging prematurely. To further improve the optimization efficiency of the PIO algorithm and prevent it from falling into a local optimal solution, this paper proposes the Hierarchical PIO Algorithm. This design is inspired by the implicit hierarchical network in the pigeon flock proposed by Nagy et al. in Nature [27], in which the relationship between leaders and followers is consistently manifested. In addition, [28] also shows that there is a hierarchical network of directional interaction in the pigeon flock, and the individual quality of the pigeon can be used as a predictor to maintain a dominant position in the group structure.

In the proposed HPIO algorithm, the hierarchical network consists of three layers. The first layer is considered to be the leaders of the pigeons of the second layer. The same is true for the relationship between the second and third layers. By letting followers imitate the leader's movement, the pigeon flock's movement decisions can be better optimized. Fig. 4 shows the conceptual diagram of the hierarchical network of pigeon flocks.

\section{1) Revised Map and Compass Operator}

Initialize the position and velocity of the pigeon flock with the population size of $N$, and the population size of each layer from the first to the third layer is denoted as $N_{1}, N_{2}, N_{3}$. The research in [27] reveals that most individuals in the bird flock tend to assume leadership roles, and the level of leadership is likely to be related to individual navigation efficiency. In the proposed HPIO algorithm, before updating the position in each iteration, the pigeon flock is re-layered according to the ranking of the individual fitness function values:

$$
\begin{aligned}
& \operatorname{rank}\left(x_{1}, x_{2}, \ldots, x_{\mathrm{N}}\right)=\left[x_{\text {rank } 1}, x_{\text {rank } 2}, \ldots, x_{\text {rank } \mathrm{N}}\right] \\
& \operatorname{rank}\left(x_{1}, x_{2}, \ldots, x_{\mathrm{N}}\right)=\left[v_{\text {rank } 1}, v_{\text {rank } 2}, \ldots, v_{\text {rank } \mathrm{N}}\right]
\end{aligned}
$$

Thus, the position and velocity of each pigeon sorted according to the individual fitness value from large to small are obtained. They are denoted as $x_{r a n k j}$ and $v_{r a n k j}$. In the process of updating the position in each iteration, the pigeon of the first layer, considered the current highest leader, flies to the historical optimal position, and other followers fly to the position where their leaders were before the update. Under this hierarchical network, all pigeons make new motion decisions at the same time, and there is no time delay in information transmission between individuals at different layers. The revised map and compass operators in the HPIO algorithm are expressed as:

While $j=1, \ldots, N_{1}$, 
TABLE II

THE PARAMETERS OF THE PV MODULE

\begin{tabular}{ccc}
\hline \hline PV module data & FG-2BTM-100 & 5S1P System \\
\hline Maximum Power $P_{\max }$ & $99.68 \mathrm{~W}$ & $498.4 \mathrm{~W}$ \\
Open circuit voltage $V_{o c}$ & $23.3 \mathrm{~V}$ & $116.5 \mathrm{~V}$ \\
Short-circuit current $I_{s c}$ & $6.4 \mathrm{~A}$ & $6.4 \mathrm{~A}$ \\
Voltage at maximum power point $V_{m p p}$ & $17.8 \mathrm{~V}$ & $89 \mathrm{~V}$ \\
Current at maximum power point $I_{m p p}$ & $5.6 \mathrm{~A}$ & $5.6 \mathrm{~A}$ \\
Temperature coefficient of $V_{o c}$ & -0.32601 & -0.35691 \\
& $(\% /$ deg.C) & $(\% / \mathrm{deg} . \mathrm{C})$ \\
Temperature coefficient of $I_{s c}$ & $0.006(\% /$ deg.C) & 0.050102 \\
& & $(\% /$ deg.C) \\
\hline \hline
\end{tabular}

TABLE III

THE PARAMETERS OF CIRCUIT COMPONENTS Specification

\begin{tabular}{cc}
\hline \hline Specification & Parameter \\
\hline Input Voltage at maximum power point $\left(V_{\text {in }}\right)$ & $89 \mathrm{~V}$ \\
Output Voltage at maximum power point $\left(V_{\text {out }}\right)$ & $223 \mathrm{~V}$ \\
Output Power of PV array at maximum power point $\left(P_{\text {out }}\right)$ & $498.4 \mathrm{~W}$ \\
Switching Frequency $\left(f_{s}\right)$ & $20 \mathrm{kHz}$ \\
$L$ & $2 \mathrm{mH}$ \\
$C_{\text {in }}$ & $1 \mu \mathrm{F}$ \\
$C_{\text {out }}$ & $10 \mu \mathrm{F}$ \\
\hline \hline
\end{tabular}

$$
v_{\text {rank } j}^{k}=v_{\text {rank } j}^{k-1} \cdot e^{-R \cdot k}+\text { rand } \cdot\left(x_{g b e s t}-x_{\text {rank } j}^{k-1}\right)
$$

While $j=N_{1}+1, \ldots, N_{1}+N_{2}$,

$$
v_{\text {rank } j}^{k}=v_{\text {rank } j}^{k-1} \cdot e^{-R \cdot k}+\sum_{m=1}^{N_{1}} \operatorname{rand} \cdot\left(x_{\text {rank } m}^{k-1}-x_{\text {rank } j}^{k-1}\right)
$$

While $j=N_{1}+N_{2}+1, \ldots, N$,

$$
v_{\text {rank } j}^{k}=v_{\text {rank } j}^{k-1} \cdot e^{-R \cdot k}+\sum_{m=N_{1}+1}^{N_{1}+N_{2}} \operatorname{rand} \cdot\left(x_{\text {rank } m}^{k-1}-x_{\text {rank } j}^{k-1}\right)
$$

Compared with the conventional PIO algorithm, in this operator, all the pigeons in the pigeon flock do not directly fly to the historical optimal position, but fly to different positions hierarchically. This makes the search scope further increase, so the HPIO algorithm has a stronger global search capability.

In the stage of the revised map and compass operator, this study proposes a convergence judgment method based on the degree of clustering of pigeons. Denote the position distribution width of the pigeon group as $\alpha$, the convergence condition is:

$$
\alpha^{k}<\mathrm{A}
$$

where A is the convergence factor. When (13) is satisfied, end the optimization of the current stage and enter the landmark operator.

\section{2) Consistent Landmark Operator}

When approaching the destination, the pigeon tends to use landmarks as the navigation tool. In this operator, the number of pigeons is reduced by half after each iteration, and the remaining pigeons fly to the center position $x_{\text {center }}$. The contribution of the landmark operator makes the HPIO algorithm has a strong local search ability in the later stage while having a powerful global search ability in the early stage. In this study, the convergence condition of the landmark operator stage is defined as:

$$
N^{k}=1
$$

When (14) is satisfied, obtain the historical global best position $x_{\text {gbest }}$, and the process of the proposed algorithm is completed.

\section{3) HPIO-based MPPT Method}

When the proposed MPPT method is applied, the position of the pigeon is expressed as duty cycle $D$, and the fitness function
TABLE IV

Simulation Results Of THE PROPOSED HPIO METHOD WITH DifFERENT

\begin{tabular}{|c|c|c|c|c|c|}
\hline $\begin{array}{c}\text { MPPT } \\
\text { methods }\end{array}$ & Parameter & $\begin{array}{l}\text { Tracking } \\
\text { time } T(\mathrm{~s})\end{array}$ & $\begin{array}{c}\text { Static } \\
\text { Tracking } \\
\text { efficiency } \\
\eta\end{array}$ & $\begin{array}{l}\text { Tracking } \\
\text { success } \\
\text { rate } \sigma\end{array}$ & $\begin{array}{c}\text { Dynamic } \\
\text { tracking } \\
\text { efficiency } \\
\eta_{d}\end{array}$ \\
\hline $\begin{array}{l}\text { The } \\
\text { proposed } \\
\text { HPIO } \\
\text { method }\end{array}$ & $\begin{array}{c}\mathrm{N}=5 \\
N_{1}=1 \\
N_{2}=2 \\
N_{3}=2 \\
\mathrm{R}=0.6 \\
\mathrm{~A}=0.1\end{array}$ & 0.2946 & $99.20 \%$ & $\begin{array}{l}100.0 \% \\
(56 / 56)\end{array}$ & $93.42 \%$ \\
\hline $\begin{array}{l}\text { The } \\
\text { DPSO } \\
\text { method } \\
\text { in [29] }\end{array}$ & $\begin{array}{c}\mathrm{N}=3 \\
\omega=0.4 \\
V_{\max }=0.035 \\
\varepsilon=0.07\end{array}$ & 0.4745 & $99.30 \%$ & $\begin{array}{l}98.21 \% \\
(55 / 56)\end{array}$ & $84.80 \%$ \\
\hline $\begin{array}{l}\text { The } \\
\text { MFA } \\
\text { method } \\
\text { in [30] }\end{array}$ & $\begin{array}{c}\mathrm{N}=5 \\
\beta_{0}=1 \\
\gamma=0.96 \\
\varepsilon=0.05\end{array}$ & 0.1855 & $98.07 \%$ & $\begin{array}{l}83.93 \% \\
(47 / 56)\end{array}$ & $93.06 \%$ \\
\hline $\begin{array}{l}\text { The OD } \\
\text { method } \\
\text { in [21] }\end{array}$ & $\begin{array}{c}\mathrm{N}=5 \\
\gamma=0.1 \\
\varepsilon=0.07\end{array}$ & 0.3988 & $99.31 \%$ & $\begin{array}{l}96.43 \% \\
(54 / 56)\end{array}$ & $89.06 \%$ \\
\hline $\begin{array}{c}\text { The } \\
\text { S-Jaya } \\
\text { method } \\
\text { in [31] }\end{array}$ & $\mathrm{N}=5$ & 0.3438 & $98.30 \%$ & $\begin{array}{l}94.64 \% \\
(53 / 56)\end{array}$ & $91.20 \%$ \\
\hline $\begin{array}{l}\text { The MIC } \\
\text { method } \\
\text { in [32] }\end{array}$ & $\begin{array}{c}\Delta D= \\
0.015 \\
V_{o c}=23.3 \\
V_{\max }=116.5 \\
V_{\min }=0 \\
N_{\max }=5\end{array}$ & 0.4408 & $99.29 \%$ & $\begin{array}{l}100.0 \% \\
(56 / 56)\end{array}$ & $86.65 \%$ \\
\hline $\begin{array}{l}\text { The } \\
\text { original } \\
\text { PIO } \\
\text { method }\end{array}$ & $\begin{array}{c}N=5 \\
R=0.6 \\
A=0.1\end{array}$ & 0.3034 & $99.08 \%$ & $\begin{array}{l}94.64 \% \\
(53 / 56)\end{array}$ & $92.77 \%$ \\
\hline
\end{tabular}
VALUES OF $R$

\begin{tabular}{ccccc}
\hline \hline $\begin{array}{c}\text { MPPT } \\
\text { method }\end{array}$ & Parameter & $\begin{array}{c}\text { Tracking } \\
\text { time } T(\mathrm{~s})\end{array}$ & $\begin{array}{c}\text { Tracking } \\
\text { efficiency } \\
\eta(\%)\end{array}$ & $\begin{array}{c}\text { Tracking success } \\
\text { rate } \sigma\end{array}$ \\
\hline & $R=0.40$ & 0.3184 & 98.67 & $89.29 \%(50 / 56)$ \\
& $R=0.45$ & 0.3107 & 98.63 & $89.29 \%(50 / 56)$ \\
The & $R=0.50$ & 0.3080 & 98.60 & $89.29 \%(50 / 56)$ \\
proposed & $R=0.55$ & 0.2938 & 98.84 & $92.86 \%(52 / 56)$ \\
HPIO & $R=0.60$ & 0.2946 & 99.20 & $100.0 \%(56 / 56)$ \\
method & $R=0.65$ & 0.2932 & 98.92 & $96.43 \%(54 / 56)$ \\
& $R=0.70$ & 0.2896 & 98.78 & $96.43 \%(54 / 56)$ \\
& $R=0.75$ & 0.2875 & 98.92 & $92.86 \%(52 / 56)$ \\
& $R=0.80$ & 0.2886 & 98.84 & $92.86 \%(52 / 56)$ \\
& $R=0.85$ & 0.2864 & 98.57 & $85.71 \%(48 / 56)$ \\
\hline \hline
\end{tabular}

TABLE V

Simulation Results OF SEVEN MPPT METHOdS UNDER 56 PSCS

is expressed as power $P$.

To use HPIO for designing MPPT, we need to determine the control variable, objective function and constraints for the optimization process. First, the control variable is chosen as the duty cycle $D$. Second, the objective function is $\max \left(P_{j}^{k}\right)$. Third, the constraints include: $\mathrm{D}_{\min } \leq D_{j}^{k} \leq \mathrm{D}_{\max }\left(\mathrm{D}_{\max }\right.$ and $\mathrm{D}_{\min }$ are chosen as 0.90 and 0.10 respectively), the maximum number of duty cycle changes is 50 .

The process of the method is as follows:

Step 1: Set the initial population number $N=5$, the number of pigeons in the first layer $N_{1}=1$, the number of pigeons in the 
second layer $N_{2}=2$, and the number of pigeons in the third layer $N_{3}=2$. The duty cycle of pigeons is initialized with uniform distribution.

Step 2: Output the duty cycle of all pigeons, measure the voltage and current of the PV system, and then calculate the power of each pigeon. Compare them with the previous historical global optimal power $P_{\text {gbest }}$ obtained, and update the historical global optimal duty cycle $D_{g b}=x_{\text {gbest }}$. Set $k=k+1$.

Step 3: Sort the pigeons by comparing the power values from large to small, thus establishing a top-down hierarchical network.

Step 4: Implement the revised map and compass operator, and use the optimization mechanism of the hierarchical network to update the duty cycle and velocity of pigeons.

Step 5: Check the first convergence condition: if the position distribution width of the pigeon flock $\alpha^{k}$ satisfies (13), jump to Step 6; otherwise, return to Step 2.

Step 6: Use the same content as in Step 2.

Step 7: Implement the landmark operator: update $N^{k}$, the center duty cycle $D_{\text {center }}^{k}=x_{\text {center }}^{k}$ and the duty cycle of the remaining pigeons.

Step 8: If the number of remaining pigeons $N^{k}$ satisfies (14), skip to Step 9; otherwise, return to Step 6.

Step 9: End and output the historical global optimal duty cycle $D_{g b}$

Furthermore, a tracking technique is introduced which switches between two MPPT modes. At the initial startup, the HPIO algorithm is activated to quickly determine the approximate location of GMPP, this process is called "Intelligent Mode"; then activate the $\mathrm{P} \& \mathrm{O}$ algorithm, so that the real-time tracking point is disturbed near the GMPP, this process is called "Disturbance Mode". The "Intelligent Mode" can be reactivated when the irradiance changes are detected with the following condition:

$$
\Delta P \%=\left|\left(P^{k}-P^{k-1}\right) / P^{k}\right|>2 \%
$$

where $P^{k}$ and $P^{k-1}$ represent the power values corresponding to the two adjacent duty cycles.

The flowchart of the proposed method is shown in Fig. 5. Fig. 6 shows the position changes of the pigeon flock during a single tracking process.

Iteration 1: Sends five initial positions of pigeons and samples the voltage and current of the PV array to calculate the corresponding power of each duty cycle. In this iteration, $D_{3}$ is $D_{g b}$.

Iteration 2: Execute revised map and compass operator of HPIO algorithm to obtain the second generation of pigeons. In this iteration, $D_{3}$ is the optimal position among the five positions again, and the position of $D_{3}$ is the same as the previous iteration.

Iteration 3: Repeat Iteration 2 and obtain the third generation of pigeons. $D_{4}$ is the current optimal position among the five positions. Since $P\left(D_{4}\right)>P\left(D_{g b}\right), D_{g b}$ is replaced by $D_{4}$.

Iteration 4: Repeat the same process as the last iteration and obtain the fourth generation. $D_{3}$ is the current optimal position. Since $P\left(D_{3}\right)=P\left(D_{g b}\right), D_{g b}$ remains unchanged.

Iteration 5: Repeat the same process as the last iteration and obtain the fifth generation. $D_{2}$ is the current optimal position. Due to $P\left(D_{2}\right)=P\left(D_{g b}\right), D_{g b}$ remains unchanged. After this iteration, (13) is satisfied. Thus, the landmark operator will be executed in next iteration.

Iteration 6: Execute the landmark operator of HPIO to obtain the sixth generation. Two pigeons with the worst positions in the previous iteration will be discarded, and the remaining three pigeons will be given new positions. By comparison, $D_{2}$ is the current optimal position. Due to $P\left(D_{2}\right)>P\left(D_{g b}\right), D_{g b}$ is replaced by $D_{2}$.

Iteration 7: In this iteration, $D_{5}$ will be discarded, $D_{2}$ and $D_{3}$ will move toward the center of themselves. Since $D_{2}$ and $D_{3}$ are almost coincident in Iteration $6, D_{g b}$ will not change after Iteration 7. Since the change process of Iteration 7 is not obvious, the process of Iteration 7 is not drawn in Fig. 6 .

After Iteration 7 , by continuing to execute the landmark operator, the number of pigeons $N$ is reduced to one. And the convergence condition in (14) is satisfied. Thus, the iterative process of the HPIO stops and $D_{g b}$ outputs as the historical global best. At this point, the HPIO algorithm has been completed.

\section{IV.SIMULATION RESULTS}

To verify the effectiveness of the proposed HPIO-based MPPT method, a $500 \mathrm{~W}$ PV system is built, as shown in Fig. 2. The PV module is FG-2BTM-100, with the parameters shown in Table II. The parameters of circuit components are listed in Table III. The simulation studies are implemented under MATLAB/Simulink.

The performance of the proposed HPIO method is compared with original PIO algorithm and four existing advanced MPPT techniques, including DPSO (Deterministic Particle Swarm Optimization) in [29], MFA (Modified Firefly Algorithm) in [30], OD (Overall Distribution) in [21], S-Jaya (cubic-spline-guided Jaya) in [31]. In addition, in order to show the comparison between the proposed method and the non-artificial intelligence-based method, MIC (Modified Incremental Conductance) in [32] is selected as the last comparison algorithm. In order to analyze the capability of different MPPT methods to deal with PSCs, it is necessary to set up multiple combinations of irradiance for simulation. The full sunshine condition is defined as receiving $G_{0}=1000 \mathrm{~W} / \mathrm{m} 2$, while the partial shading conditions receive $G_{i}=n_{i} \times G_{0} \mathrm{~W} / \mathrm{m} 2$ $\left(n_{i} \times 100 \%\right.$ of the full sunshine). Thus, the irradiance value of each PV module is $n_{i} \times 1000\left(n_{i} \in\{0.125,0.250,0.375,0.500,0.625\right.$, $0.750,0.875,1.000\})$. Based on the above analysis, $56\left(C_{8}^{5}\right)$ PSCs can be obtained. The average performances of the seven MPPT methods are obtained by combining the simulation results under 56 PSCs.

\section{A. Performance Evaluation}

To accurately evaluate the performance, the performance of the MPPT methods is compared through three indicators as follows:

\section{1) Static Tracking Efficiency $\eta$}

$$
\eta=\frac{P_{m}}{P_{M P P}} \times 100 \%
$$

where $P_{m}$ is the final maximum output power, and $P_{M P P}$ is the 
This article has been accepted for publication in a future issue of this journal, but has not been fully edited. Content may change prior to final publication. Citation information: DOI10.1109/TIE.2021.3137595,

TABLE VI

Simulation Results OF SEVEn MPPT Methods Under FOUR CASES

\begin{tabular}{|c|c|c|c|c|c|c|c|}
\hline Cases & MPPT method & $\begin{array}{l}\text { Tracking voltage } \\
\text { (V) }\end{array}$ & $\begin{array}{l}\text { Tracking power } \\
\text { (W) }\end{array}$ & $\begin{array}{l}\text { Tracking } \\
\text { time } T(\mathrm{~s})\end{array}$ & $\begin{array}{l}\text { Static Tracking } \\
\text { efficiency } \eta(\%)\end{array}$ & $\begin{array}{c}\text { Dynamic } \\
\text { Tracking } \\
\text { efficiency } \eta_{d}(\%)\end{array}$ & $\begin{array}{l}\text { Tracking } \\
\text { GMPP } \\
\text { success? }\end{array}$ \\
\hline \multirow{6}{*}{$\begin{array}{c}\text { Case } \\
1\end{array}$} & The proposed HPIO method & 88.88 & 495.8 & 0.25 & 99.48 & 91.01 & Yes \\
\hline & The DPSO method in [29] & 88.88 & 495.8 & 0.45 & 99.48 & 75.15 & Yes \\
\hline & The MFA method in [30] & 94.41 & 479.2 & 0.20 & 96.15 & 90.60 & No \\
\hline & The OD method in [21] & 89.31 & 495.7 & 0.40 & 99.46 & 85.78 & Yes \\
\hline & The S-Jaya method in [31] & 88.43 & 495.8 & 0.35 & 99.48 & 90.31 & Yes \\
\hline & The original PIO method & 88.88 & 495.8 & 0.30 & 99.48 & 90.55 & Yes \\
\hline \multirow{7}{*}{$\begin{array}{c}\text { Case } \\
2\end{array}$} & The proposed HPIO method & 98.23 & 292.1 & 0.30 & 99.21 & 93.45 & Yes \\
\hline & The DPSO method in [29] & 98.23 & 292.1 & 0.45 & 99.21 & 82.47 & Yes \\
\hline & The MFA method in [30] & 98.56 & 292.1 & 0.20 & 99.21 & 95.62 & Yes \\
\hline & The OD method in [21] & 98.56 & 292.1 & 0.40 & 99.21 & 91.68 & Yes \\
\hline & The S-Jaya method in [31] & 100.40 & 288.6 & 0.35 & 98.02 & 90.30 & Yes \\
\hline & The MIC method in [32] & 98.56 & 292.1 & 0.45 & 99.21 & 78.20 & Yes \\
\hline & The original PIO method & 98.23 & 292.1 & 0.30 & 99.21 & 92.62 & Yes \\
\hline \multirow{5}{*}{$\begin{array}{c}\text { Case } \\
3\end{array}$} & The MFA method in [30] & 75.38 & 280.1 & 0.20 & 99.35 & 94.67 & Yes \\
\hline & The OD method in [21] & 75.38 & 280.1 & 0.40 & 99.35 & 89.08 & Yes \\
\hline & The S-Jaya method in [31] & 75.73 & 280.2 & 0.35 & 99.38 & 91.72 & Yes \\
\hline & The MIC method in [32] & 75.00 & 279.7 & 0.41 & 99.21 & 79.34 & Yes \\
\hline & The original PIO method & 75.00 & 279.7 & 0.30 & 99.21 & 92.19 & Yes \\
\hline \multirow{7}{*}{$\begin{array}{c}\text { Case } \\
4\end{array}$} & The proposed HPIO method & 34.86 & 172.1 & 0.30 & 99.55 & 90.65 & Yes \\
\hline & The DPSO method in [29] & 33.82 & 172.1 & 0.50 & 99.55 & 75.14 & Yes \\
\hline & The MFA method in [30] & 37.59 & 155.2 & 0.20 & 89.78 & 83.68 & No \\
\hline & The OD method in [21] & 34.62 & 172.3 & 0.40 & 99.67 & 83.48 & Yes \\
\hline & The S-Jaya method in [31] & 34.10 & 172.3 & 0.35 & 99.67 & 89.59 & Yes \\
\hline & The MIC method in [32] & 34.61 & 172.3 & 0.43 & 99.67 & 77.17 & Yes \\
\hline & The original PIO method & 34.78 & 172.1 & 0.30 & 99.55 & 87.87 & Yes \\
\hline
\end{tabular}

theoretical value of the maximum output power.

2) Tracking Time $T$

$T$ is defined as the time for the PV system to reach a stable output. It can be measured by the number of sampling cycles.

3) Tracking Success Rate $\sigma$

$$
\sigma=\frac{N_{s t}}{N_{s}} \times 100 \%
$$

where $N_{s t}$ is the number of successful tracking times, and $N_{s}$ is the number of simulated times.

The condition for judging the tracking success of the MPPT method is successfully tracking the peak where the GMPP is located and meets the following conditions:

$$
\begin{gathered}
\eta \geq 95 \% \\
\frac{\left|V_{m}-V_{M P P}\right|}{V_{M P P}} \leq 0.03
\end{gathered}
$$

where $V_{m}$ is the final output voltage of the PV system, and $V_{M P P}$ is the theoretical value of the output voltage at MPP.

\section{4) Dynamic Tracking Efficiency $\eta_{d}$}

$$
\eta_{d}=\frac{P_{\text {ave }}}{P_{M P P}} \times 100 \%
$$

where $P_{\text {ave }}$ is defined as the average output power of the PV system using the MPPT algorithm for a period of $T_{\text {ave }}$ seconds after starting the MPPT algorithm. In this research, $T_{\text {ave }}$ is selected as $0.6 \mathrm{~s}$.

\section{B. Determination of Parameters for HPIO}

It can be seen from (10)-(12) that the value of $R$ needs to be determined for the proposed method. In this study, $R$ is determined based on a large number of simulation results. First, based on preliminary simulation tests, it can be found that when the value of $R$ is in the range of 0.40 to 0.85 , the proposed HPIO method can realize MPPT with good results. To further determine the optimal value of $R, R$ is set to 10 values $(0.40$, $0.45,0.50,0.55,0.60,0.65,0.70,0.75,0.80,0.85)$, and ten HPIO methods with these different $R$ values are tested under 56 PSCs. Table IV shows the average performances of ten HPIO methods with different $R$ under 56 PSCs. It can be seen that as the value of $R$ increases, the tracking time gradually shortens. When the value of $R$ is 0.60 , the proposed HPIO method obtains the optimal tracking success rate and tracking efficiency. Based on the above analysis, the value of $R$ is finally selected as 0.60 .

In the proposed HPIO MPPT method, the selection of parameter A needs to be adjusted according to the number $N_{s}$ of series connected modules. In order to ensure that the flock of pigeons has gathered on the peak where the GMPP is located when the first convergence condition is met, the setting formula of $\mathrm{A}$ is proposed in this research, namely:

$$
\mathrm{A}<0.5 \frac{1}{\mathrm{~N}_{\mathrm{s}}}
$$

The coefficient " 0.5 " is set to ensure that " $\mathrm{A}$ " can be used as the duty cycle distribution width to determine the first stage of convergence. Therefore, the value of A should be set as 0.10 .

\section{Comparative Simulated Results under 56 PSCs}

Table V shows the parameters of the seven MPPT methods and the average performance results under 56 PSCs. $\varepsilon$ represents the convergence threshold of the three MPPT methods for comparison. When the difference between all duty cycles is less than $\varepsilon$, the MPPT algorithm ends and outputs the global best duty cycle $D_{g b}$. It can be seen that the DPSO method has a slow convergence speed and exhibits the lowest dynamic tracking efficiency. However, it has a strong shadow tracking ability to achieve successful tracking. The MFA method is easy to obtain a solution prematurely, thus its ability to deal with shadows is 

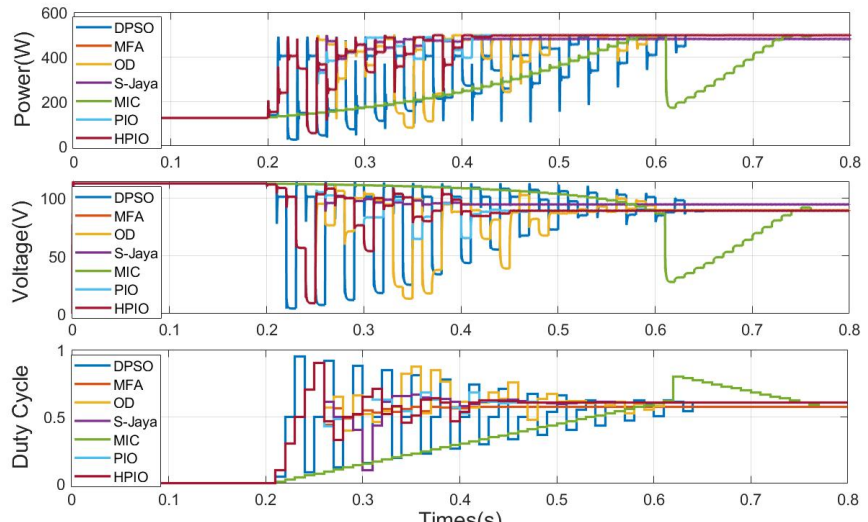

Fig. 7. The output waveforms during the tracking process in Case 1.
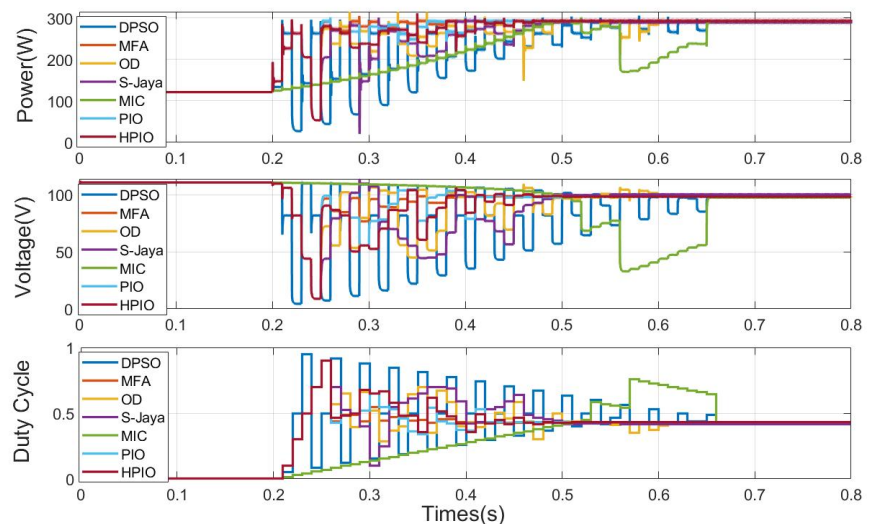

Fig. 8. The output waveforms during the tracking process in Case 2.

weak and easily leads to tracking failure. The OD method can complete the tracking process in a shorter time compared with the DPSO method, and it has a high tracking success rate while its dynamic performance is also better. The S-Jaya method shows good dynamic tracking performance. However, its static tracking efficiency is not well, and does not well achieve the requirement of accurate tracking to GMPP. Different from the above swarm-intelligence-based algorithm, the MIC method realizes intelligent scanning to the PV characteristic curve by using the relevant parameters of the PV array. Since its principle of tracking is based on the scanning method, it takes a long tracking time and has poor dynamic performance, but it has a high tracking success rate. Compared with the S-Jaya method, the original PIO method takes shorter tracking time and has a more comprehensive tracking performance. But compared with OD, DPSO and MIC methods, this method still has room for improvement in static tracking efficiency. Compared with the four advanced AI-based and one non-AI-based MPPT techniques above and the original PIO method, the proposed HPIO method has the highest tracking success rate, reaching $100 \%$, and has the highest dynamic tracking efficiency. Compared with the original PIO method, the tracking performance of the proposed method has overall been further improved. Furthermore, the proposed HPIO method has a fast convergence speed, second only to the MFA method.

Although the static tracking efficiency of the proposed HPIO method is slightly lower than that of the OD, DPSO and MIC methods, due to its powerful shadow tracking ability, superior dynamic tracking efficiency and fast convergence speed, the proposed HPIO method exhibits the most superior
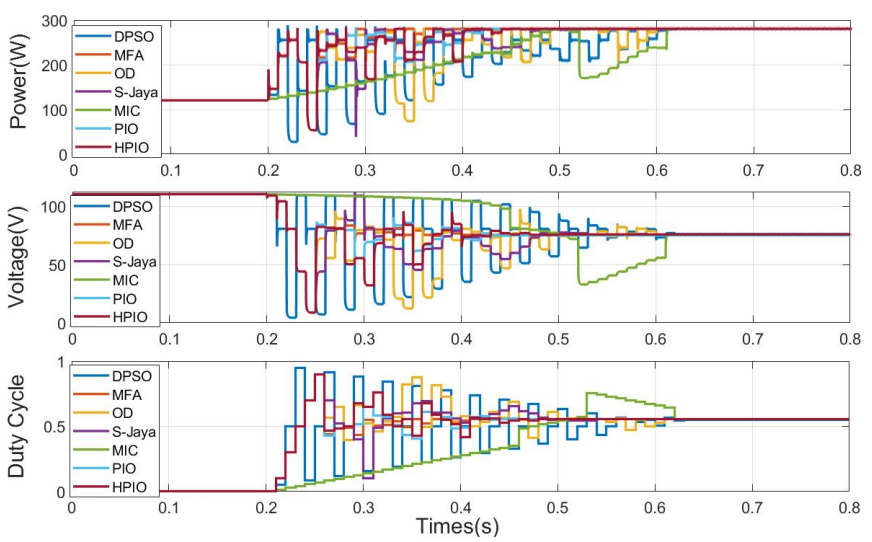

Fig. 9. The output waveforms during the tracking process in Case 3.
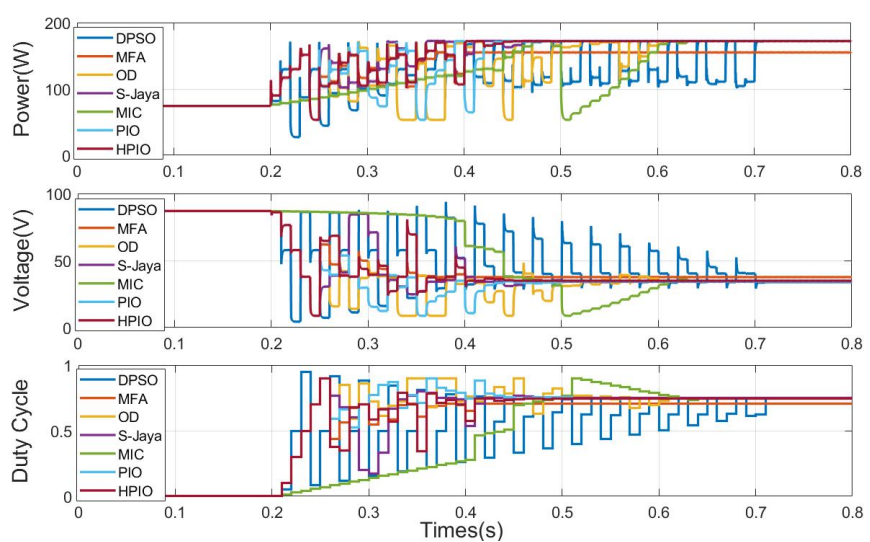

Fig. 10. The output waveforms during the tracking process in Case 4.

comprehensive tracking performance among the seven MPPT methods. The proposed HPIO method can achieve superior tracking results in a shorter time and then output the historical optimal power point. According to the MPPT process proposed in this paper, the above seven MPPT methods are implemented in the first mode-"Intelligent Mode". Next, the PV system enters the second mode-"Disturbance Mode". With the regular disturbance of the duty cycle near the GMPP position, it continues to obtain higher tracking efficiency. The above analysis shows that the proposed HPIO method has excellent performances in dealing with complex PSCs.

\section{Specific Cases Analysis}

Next, Table VI shows the simulation results of the seven MPPT methods under four different cases given in Table I and Fig. 3. Later, we also give the results of the dynamic tracking test.

1) Case 1: The output waveforms during the tracking process of the seven MPPT methods are shown in Fig. 7. It can be observed that the proposed HPIO method completes tracking within $0.25 \mathrm{~s}$, and the static tracking efficiency reaches $99.48 \%$; and the dynamic tracking efficiency reaches $91.01 \%$, the highest among the seven MPPT methods. The DPSO, MIC, S-Jaya and original PIO method complete the tracking within $0.45 \mathrm{~s}, 0.56 \mathrm{~s}, 0.35 \mathrm{~s}$ and $0.3 \mathrm{~s}$ respectively. Their static tracking efficiency also reach $99.48 \%$. The MFA method only takes $0.2 \mathrm{~s}$ to complete the tracking, but does not track to the location near GMPP, the static tracking efficiency is only $96.15 \%$. The OD method completes the tracking within $0.4 \mathrm{~s}$, and the static tracking efficiency reaches $99.46 \%$. Due to the long tracking 
time, DPSO, OD and MIC methods show a low dynamic tracking efficiency while the dynamic tracking efficiency of the other four methods all reach more than $90 \%$. Though the MFA method has the fastest tracking speed, it fails to track GMPP in this case, indicating that the MFA method lacks certain search ability.

2) Case 2: It can be seen from Fig. 8 that the HPIO, DPSO, MFA, OD, MIC and original PIO methods complete the tracking within $0.3 \mathrm{~s}, 0.45 \mathrm{~s}, 0.2 \mathrm{~s}, 0.4 \mathrm{~s}, 0.45 \mathrm{~s}$ and $0.3 \mathrm{~s}$ respectively, and the six MPPT methods finally achieve the same static tracking efficiency of $99.21 \%$. The S-Jaya method completes the tracking within $0.35 \mathrm{~s}$, and the static tracking efficiency only reaches $98.02 \%$. In this case, the MFA method shows the highest dynamic tracking efficiency of $95.62 \%$; the HPIO method is second only with $93.45 \%$; the dynamic tracking efficiency of MPPT methods except DPSO and MIC reach more than $90 \%$. Obviously, the proposed HPIO method shows the tracking speed and dynamic tracking efficiency second only to the MFA method, and its static tracking efficiency is no less than that of the other six MPPT methods.

3) Case 3: It can be seen from Fig. 9 that the proposed HPIO and S-Jaya method complete the tracking within $0.3 \mathrm{~s}$ and $0.35 \mathrm{~s}$ respectively, and the static tracking efficiency reaches $99.38 \%$. The DPSO, MFA and OD methods complete tracking within $0.42 \mathrm{~s}, 0.2 \mathrm{~s}$ and $0.4 \mathrm{~s}$ respectively. These three MPPT methods finally achieve the same static tracking efficiency of $99.35 \%$. The MIC and original PIO method complete the tracking within $0.41 \mathrm{~s}$ and 0.30 s respectively, and their static tracking efficiency reach $99.21 \%$. In this case, the MFA method shows the highest dynamic tracking efficiency of $94.67 \%$; the HPIO method is second only with $92.87 \%$; the dynamic tracking efficiency of MPPT methods except DPSO, OD and MIC reach more than $90 \%$. The proposed HPIO method still shows the tracking speed and dynamic tracking efficiency second only to the MFA method and the highest static tracking efficiency among the six MPPT methods.

4) Case 4: It can be observed from Fig. 10 that the proposed HPIO and original PIO methods complete tracking within $0.3 \mathrm{~s}$, and the static tracking efficiency reaches $99.55 \%$. The DPSO method still does not converge within $0.50 \mathrm{~s}$, and the maximum number of samplings is reached at this time so that it ends tracking and outputs the optimal point. The MFA method only needs 0.2 s to complete the tracking; however, due to the overly fast convergence, the tracked position deviates from the GMPP, the static tracking efficiency is only $89.78 \%$; thus, it fails to track the GMMP. The OD, S-Jaya and MIC method complete the tracking within $0.4 \mathrm{~s}, 0.35 \mathrm{~s}$ and $0.43 \mathrm{~s}$ respectively, and the static efficiency reaches $99.67 \%$. The proposed HPIO method shows the highest dynamic tracking efficiency of $90.65 \%$; while the dynamic tracking efficiency of other MPPT methods fail to reach $90 \%$. In Case 4, the MFA method fails to realize MPPT, again indicating that its shadow tracking ability is also lacking.

5) Dynamic Tracking Test: In order to verify the practicability of the MPPT design based on the two modes proposed in this paper for long-term tracking, dynamic tracking tests are carried out on the seven MPPT methods under the simulation environment of dynamic changes in irradiance and continuous switching of PSCs. The seven MPPT methods are tested using the MPPT framework based on two modes. Based
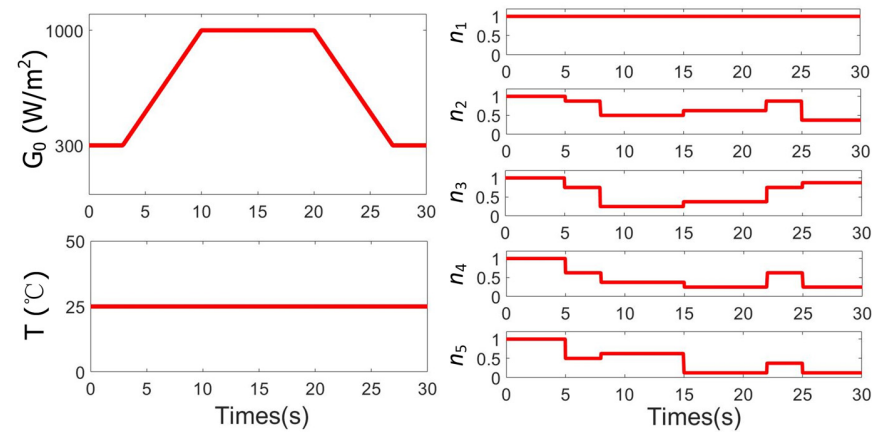

Fig. 11. The test profiles for dynamic tracking test.

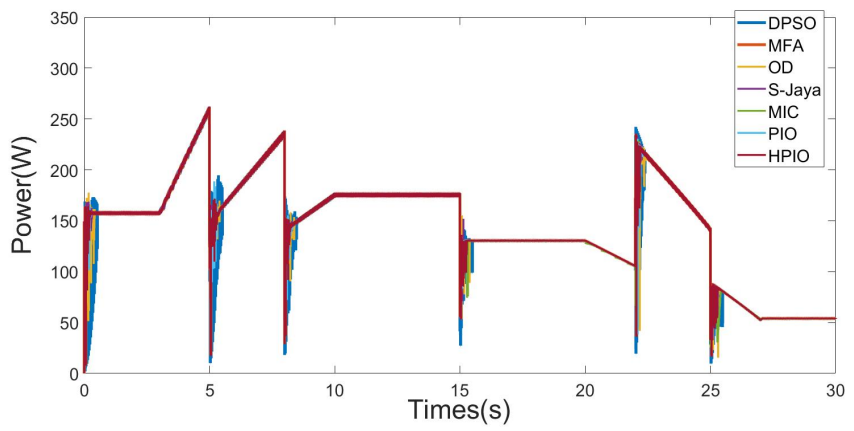

Fig. 12. The power waveforms during the dynamic tracking test.

on the EN50530 standard, a 30\%-100\% ramp with a slope of $100 \mathrm{~W} / \mathrm{m}^{2}$ and a dwell time of $10 \mathrm{~s}$ is selected as the irradiance curve. It should be noted that the percentage specification of irradiance is related to STC, and $100 \%$ corresponds to 1000 $\mathrm{W} / \mathrm{m}^{2}$ at $25{ }^{\circ} \mathrm{C}$. The test profiles, including the selected irradiance curve and the percentage $n_{i}$ of the irradiance received by each PV panel in real time, are shown in Fig. 11. And Fig. 12 shows the power waveforms of seven MPPT methods in the current test environment. It can be seen that all MPPT methods still keep track of GMPP during the whole process of changing environmental conditions. In the proposed MPPT framework based on two modes, the intelligent MPPT algorithm is restarted to find a new GMPP so that effectively dealing with PSC switching.

6) Summary: Based on the analysis of the above four cases, it can be seen that the proposed HPIO method still has perfect shadow tracking ability and high static and dynamic tracking efficiency in a relatively short tracking time, and has the best overall performance among the seven MPPT methods. It indicates that the proposed HPIO method has superior performance in dealing with complex PSCs.

Through the change of duty cycle and voltage waveform of the proposed HPIO method, it can be seen that from the initial distribution to the end of the first stage of optimization, the change trend of duty cycle and voltage is relatively gentle due to the stratified movement, and will not gather to the current global optimal power point too quickly, thus realizing the precise search for various locations within the initial distribution range during the process of multiple iterations. In the second stage of optimization, due to the reduction of population size, the tracking time is very short. During this period, the duty cycle and voltage fluctuate around the global optimal power point to ensure that GMPP could be found. 


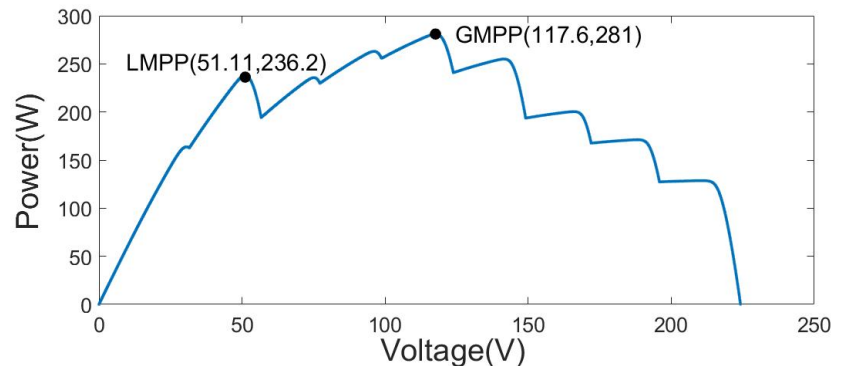

Fig. 13. The P-V characteristic curve under complex PSC with multiple clusters.
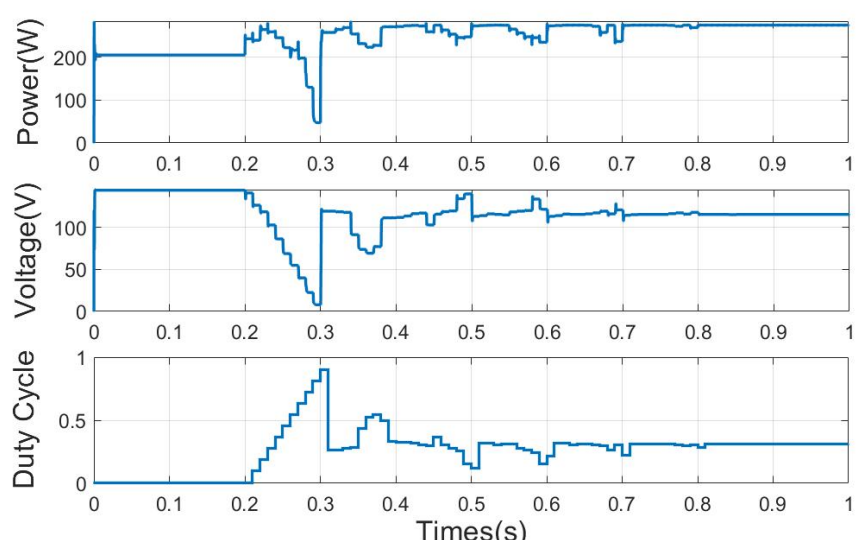

Fig. 14. The output waveforms under PSC with multiple clusters.
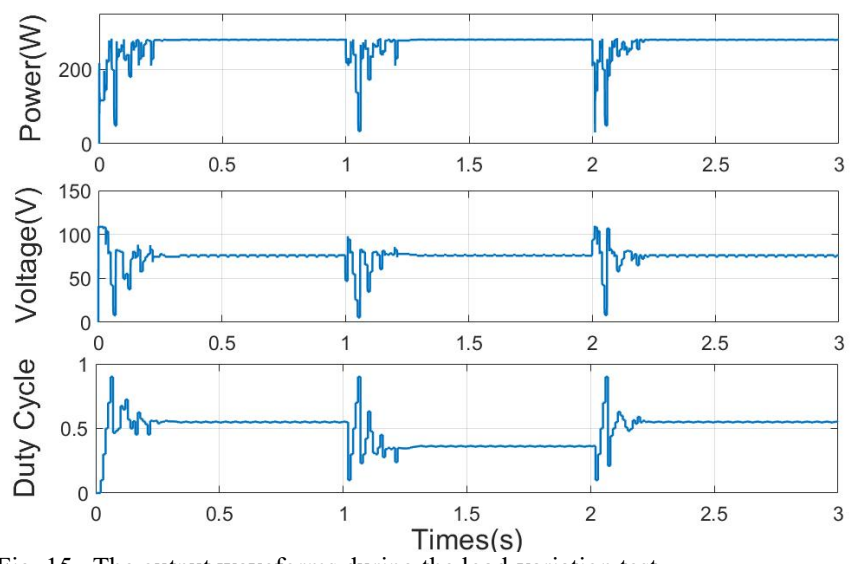

Fig. 15. The output waveforms during the load-variation test.

\section{E. Complex PSC with Multiple Clusters}

Kermadi et al. [13] discussed that complex PSC may form multiple clusters, and each cluster has its own middle peak (MHP). The P-V curve with multiple MHP brings difficulties to tracking MPP. In order to verify that the proposed HPIO method is still valid under PSC with multiple clusters, the following simulation results are given. The $\mathrm{P}-\mathrm{V}$ characteristic curve of the PSC selected for the simulation is shown in Fig. 13. It can be seen that the curve has two MHPs, and GMPP is at the middle of the curve. In this curve, $V_{M P P}$ is $117.6 \mathrm{~V}$, and $P_{M P P}$ is 281.0W.

Fig. 14 shows the output waveforms of the proposed HPIO method in the current shading pattern. It can be observed from Fig. 14 that the proposed HPIO complete tracking within $0.6 \mathrm{~s}$, $V_{m}$ is $115.3 \mathrm{~V}$ and $P_{m}$ is $275.0 \mathrm{~W}$. The static tracking efficiency reaches $97.86 \%$, and the dynamic tracking efficiency within

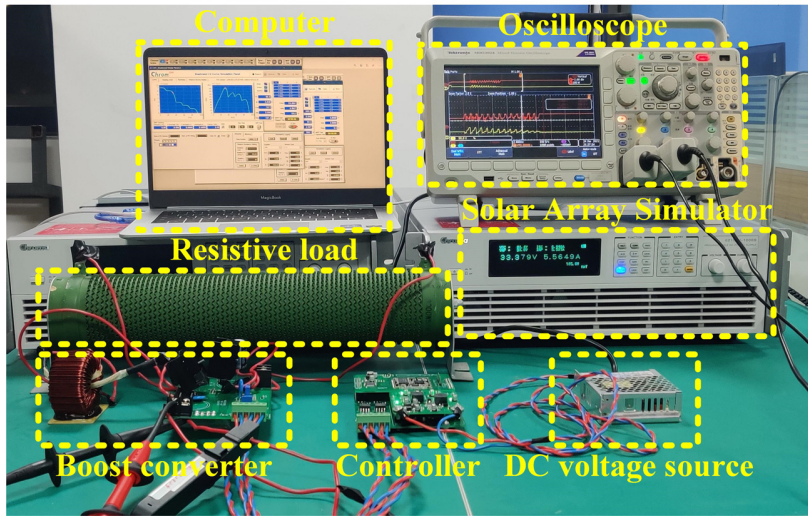

Fig. 16. The composition of the experimental platform.

TABLE VII

THE PARAMETERS OF CIRCUIT COMPONENTS

\begin{tabular}{cc}
\hline \hline Specification & Parameter \\
\hline Input Voltage at MPP $\left(V_{\text {in }}\right)$ & $84.2 \mathrm{~V}$ \\
Output Power of PV array at MPP $\left(P_{\text {out }}\right)$ & $507.0 \mathrm{~W}$ \\
Switching Frequency $\left(f_{s}\right)$ & $20 \mathrm{kHz}$ \\
$L$ & $5 \mathrm{mH}$ \\
$C_{\text {in }}$ & $100 \mu \mathrm{F}$ \\
$C_{\text {out }}$ & $47 \mu \mathrm{F}$ \\
\hline \hline
\end{tabular}

0.8 s reaches $92.65 \%$. Therefore, it can be concluded that the proposed HPIO method can still successfully track GMPP under complex PSC with multiple clusters.

\section{F. Load Changes under PSCs}

In practical applications, in addition to constantly changing irradiance conditions, load changes are also important external conditions that affect the dynamic operation of the PV system. In order to verify the effectiveness of the proposed MPPT design based on the two modes in response to load changes, load-varying tests are carried out on the proposed HPIO method.

In this test, select Case 3, which is given in Table I and Fig. 3, as the PSC. $V_{M P P}$ is $76.38 \mathrm{~V}$, and $P_{M P P}$ is $281.9 \mathrm{~W}$. Fig. 15 shows the output waveforms of the proposed HPIO method during the load-variation test. The initial load value is $\mathrm{R}=100 \Omega$. When $t=0 \mathrm{~s}$, the MPPT method is started and GMPP is successfully traced. When $t=1 \mathrm{~s}$, the load becomes $\mathrm{R}=50 \Omega$; therefore, the controller detects the power fluctuation caused by the load change, and restarts the MPPT method. When $t=2 \mathrm{~s}$, the load becomes $\mathrm{R}=100 \Omega$; therefore, the MPPT method is restarted again. According to the results shown in Fig. 15, it can be seen that the load change conditions will not reduce the tracking performance of the proposed MPPT design based on two modes.

\section{EXPERIMENTAL RESULTS AND DISCUSSIONS}

The experimental platform consists of a solar array simulator (Chroma A62028), a boost converter, a load, a control circuit, an oscilloscope (Tektronix MDO 3024), as shown in Fig. 16. The parameters are shown in Table VII. The proposed HPIO MPPT method and the other three MPPT methods for comparison are implemented based on MCU RT1052 with 528 MHz clock, 32 MB flash memory, 1 MB SRAM and ADC chip AD7606 with the resolution of 16 bits. Four cases given in Fig. 3 are loaded into the solar array simulator to obtain the P-V and I-V curves under the four cases, shown in Fig. 17. 

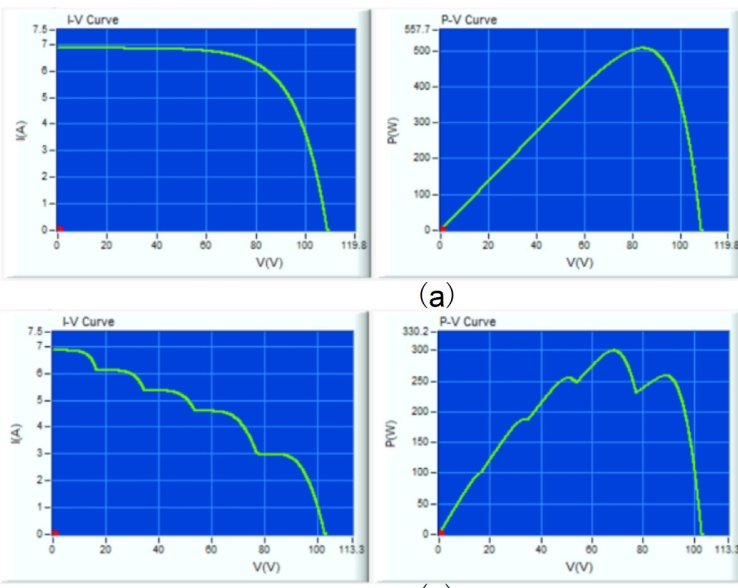

(a)

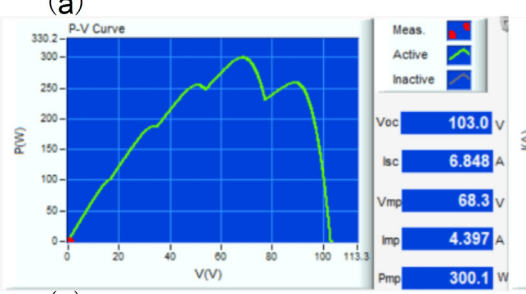

(c)
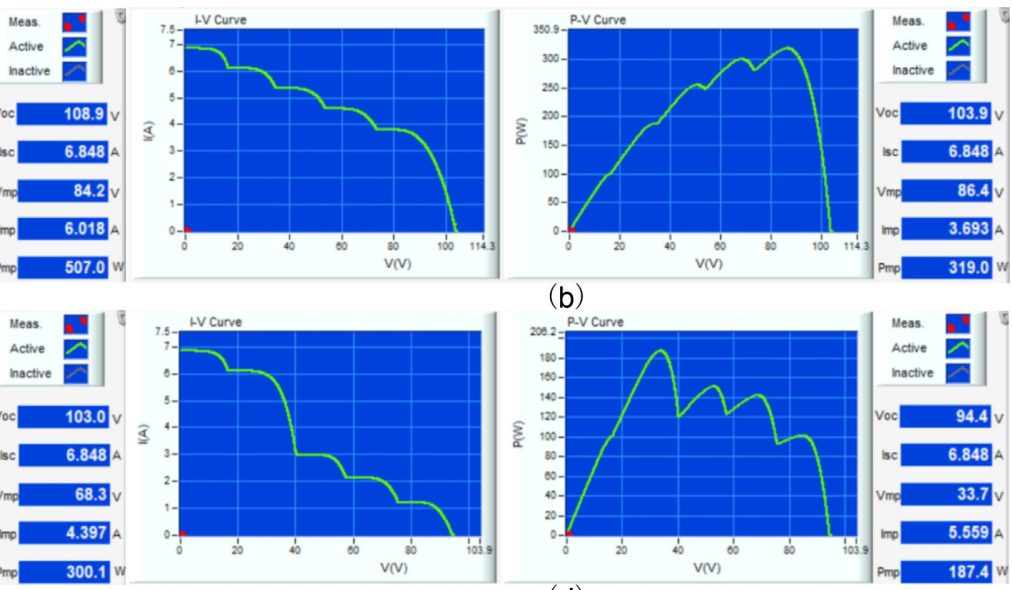

(d)

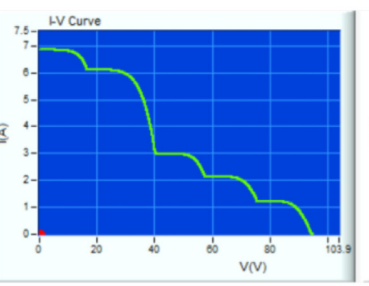

(b) Case 2. (c) Case 3. (d) Case 4.
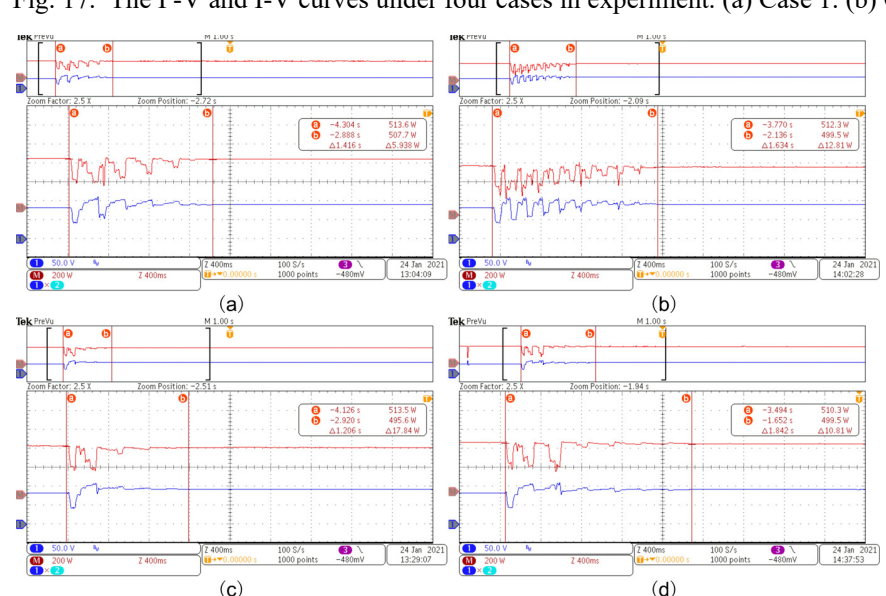

(d)

Fig. 18. The output power and output voltage waveforms under Case 1. (a) Proposed HPIO method. (b) DPSO method. (c) MFA method. (d) OD method.
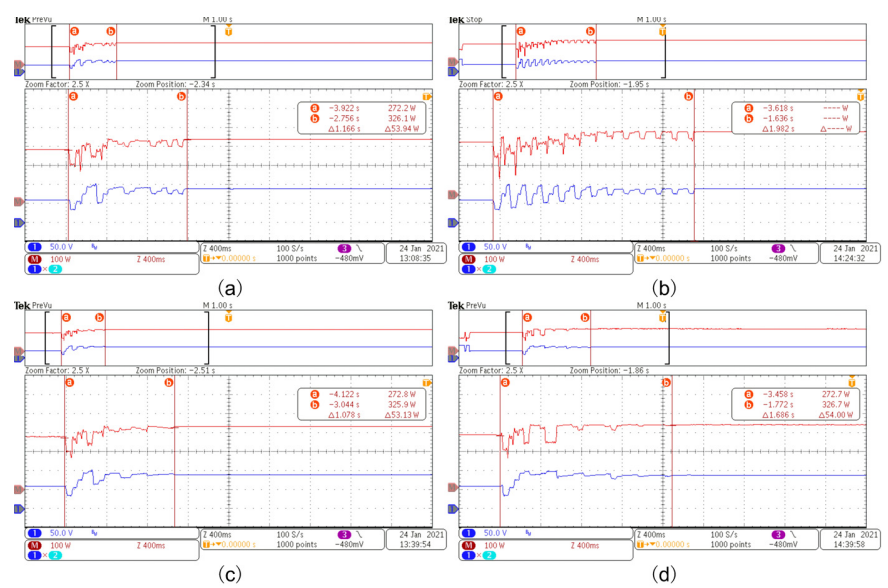

Fig. 19. The output power and output voltage waveforms under Case 2. (a) Proposed HPIO method. (b) DPSO method. (c) MFA method. (d) OD method.

The input voltage and inductor current of the boost converter pass through the voltage-voltage and current-voltage conversion circuit, and after being sampled by AD7606, they are transmitted to the program that implements the MPPT method. The MPPT methods adjust the position of the tracking point by outputting a duty cycle to the boost converter, thereby achieving MPPT technology. Due to the need to consider the response speed of the circuit in the steady state during each sampling process, the sampling time, which is from the
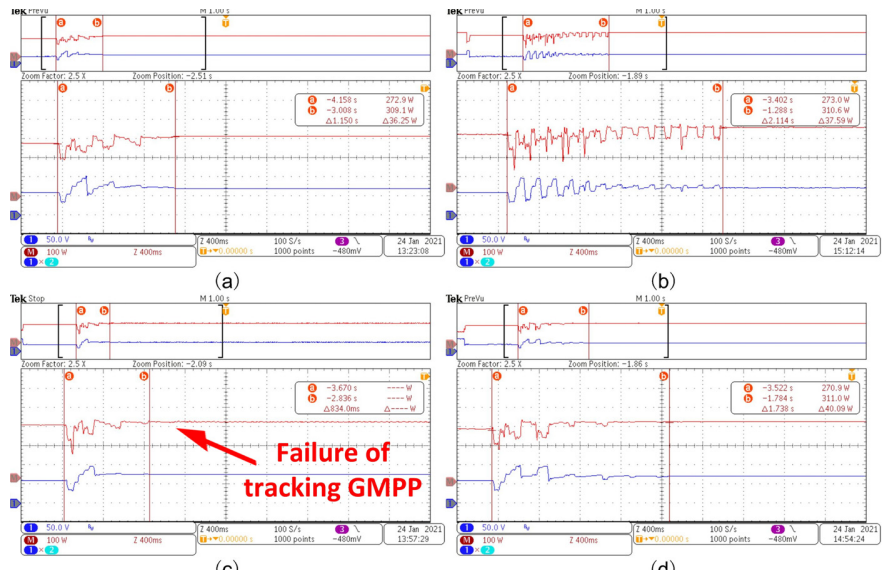

(d)

Fig. 20. The output power and output voltage waveforms under Case 3. (a) Proposed HPIO method. (b) DPSO method. (c) MFA method. (d) OD method.
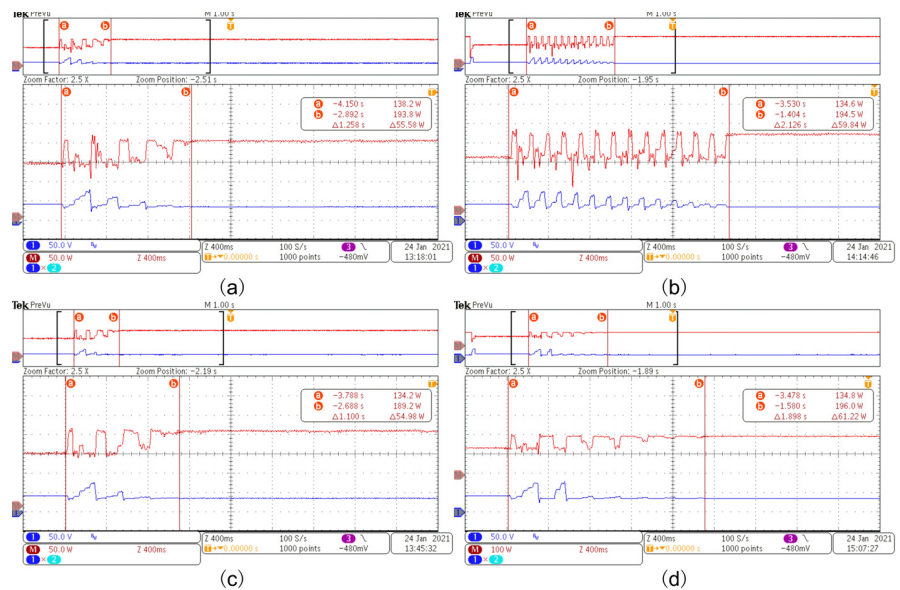

Fig. 21. The output power and output voltage waveforms under Case 4. (a) Proposed HPIO method. (b) DPSO method. (c) MFA method. (d) OD method.

controller output duty cycle to the collection of the voltage and current values, is set to $0.055 \mathrm{~s}$ to meet the technical requirements of MPPT. In this section, three AI-based MPPT methods, that is, DPSO, MFA and OD, are selected to compare the tracking performance with the proposed HPIO method in the same experimental environment. Considering that the S-Jaya method exhibits weak tracking performance, the MIC method requires additional parameters of the PV array so that it causes inconvenience to the practical application, and the 
original PIO method is the prototype of the HPIO method, these three methods are not selected as the comparison method. In the experiment, the duty cycle range of all MPPT methods is set at $[0.1,0.70]$. The other parameters of the four MPPT methods in the experiment are the same as the other parameters of the simulation research.

1) Case 1: Fig. 18 shows the output power and output voltage waveforms of the PV system. For the proposed HPIO method, it takes $1.416 \mathrm{~s}$ to complete the MPPT process. After the power stabilizes, the measured tracking power is $500.7 \mathrm{~W}$, and the efficiency reaches $98.76 \%$. For the DPSO method, MFA method and OD method, the tracking time reaches $1.634 \mathrm{~s}$, $1.206 \mathrm{~s}$ and $1.842 \mathrm{~s}$ respectively, and the tracking efficiency also reaches $97.75 \%$. 96.79\% and 97.73\%. Compared with other three MPPT methods, the proposed HPIO method has a higher tracking efficiency. Although the MFA method has the fastest tracking speed, its tracking efficiency is $1.97 \%$ lower than that of the proposed HPIO method.

2) Case 2: As observed from Fig. 19, for the proposed HPIO method, it takes $1.166 \mathrm{~s}$ to complete the MPPT, and the tracking efficiency reaches $99.81 \%$. For the DPSO method, MFA method and OD method, the tracking time is $1.982 \mathrm{~s}, 1.078 \mathrm{~s}$ and $1.686 \mathrm{~s}$ respectively, and the tracking efficiency reaches $99.75 \%$ $99.69 \%$ and $99.72 \%$. In this case, the four MPPT methods successfully track to the power point near GMPP. The proposed HPIO method and MFA method have significantly faster tracking speeds compared with the other two methods.

3) Case 3: As seen from Fig. 20, for the proposed HPIO method, it takes 1.150 s to complete the MPPT process, and the tracking efficiency reaches $99.80 \%$. For the DPSO method, MFA method and OD method, the tracking time is $2.114 \mathrm{~s}$, $0.834 \mathrm{~s}$ and $1.738 \mathrm{~s}$ respectively, and the tracking efficiency reaches $99.83 \%$. 93.74\% and $99.87 \%$ as well. The MFA method can only track to the power point of $7.82 \%$ Voc away from GMPP due to the fast convergence, which leads to the failure of tracking GMPP. Moreover, due to the slow convergence of the DPSO method, the MPPT ends when the maximum number of samples is reached.

4) Case 4: As observed from Fig. 21, for the proposed HPIO method, it takes $1.258 \mathrm{~s}$ to complete the MPPT process with the tracking efficiency of $98.72 \%$. For the DPSO method, MFA method and OD-PSO method, the tracking time is $2.126 \mathrm{~s}$, $1.100 \mathrm{~s}$ and $1.898 \mathrm{~s}$ respectively, and the tracking efficiency reaches $98.72 \%, 98.19 \%$ and $98.88 \%$ too. Similarly, the proposed HPIO and MFA have significantly faster tracking speed than the other two MPPT methods. MFA has the fastest tracking speed, but its tracking efficiency is slightly lower among the four MPPT methods. Due to the slow convergence, the DPSO method completes the MPPT with the slowest tracking speed.

5) Summary: It can be seen that by comprehensively considering multiple indicators of tracking success rate, tracking efficiency, and tracking speed, the proposed HPIO method shows better tracking performance than the DPSO method, the MFA method and the OD method. The proposed HPIO method solves the problem that the rapid convergence of the MFA method may fall into LMPP, and also solves the problem that the convergence speed of the DPSO method and the OD-PSO method is not fast enough for foreseeable practical applications. In addition, the tracking performance of the four
MPPT methods tested under the four cases in the experiment is similar to the results obtained by the simulation, which further verifies the accuracy of this research.

As the attention to the research on PV systems continues to increase, the need to solve the problem of dust accumulation has gradually emerged. The research in [33] shows that dust accumulation will cause a decrease in voltage and current, while the drop in the open voltage can be ignored and the reduction in the output current is the main reason for the power drop. It will also reduce the inertia against the variation in the P-V and I-V characteristic curves, thus adding certain difficulties to the realization of MPPT technology. In a well-ventilated installation environment, the degree of dust accumulation on each PV panel is the same, which will not directly lead to the production of PSC. However, in the actual production and operation process, the degree of dust accumulation on each panel may be different due to the influence of the terrain around the installation environment. At this time, even if the PV system receives uniform illumination, there may be multiple power extreme points on the actual P-V characteristic curve. The existing MPPT method that can track GMPP under PSCs can also cope with the problem of dust accumulation to a certain extent.

\section{VI.CONCLUSION}

In this paper, a hierarchical pigeon-inspired optimization method is proposed for efficient and fast maximum power point tracking of PV system. In order to strengthen the global search capability, a hierarchical network in the pigeon flock is constructed to revise the map and compass operator of the original PIO algorithm to ensure superior tracking efficiency and tracking success rate. At the same time, the landmark operator can also ensure fast convergence to save tracking time. On this basis, a tracking mechanism that switches between "Intelligent Mode" and "Disturbance Mode" is proposed. With these two modes, not only can the problem of searching for GMPP under PSCs be effectively solved, but also long-term efficient dynamic tracking can be maintained. Comparative simulated results with 56 PSCs and experimental results under four typical irradiation cases verify the excellent tracking performance of the proposed HPIO method as compared with typical existing advanced MPPT techniques. Simulated results of dynamic tracking test also verify the effectiveness of the proposed MPPT framework based on two modes for practical applications. The proposed HPIO method effectively achieves a good balance between the two indicators of tracking efficiency and tracking speed in MPPT. To increase the practical application of the method further, it is important to carry out more research to deal with dust accumulation in future work too.

\section{REFERENCES}

[1] J. Ahmed and Z. Salam, "An accurate method for MPPT to detect the partial shading occurrence in a PV system," IEEE Transactions on Industrial Informatics, vol. 13, no. 5, pp. 2151-2161, Oct. 2017.

[2] N. Femia, G. Petrone, G. Spagnuolo and M. Vitelli, "Optimization of perturb and observe maximum power point tracking method," IEEE Transactions on Power Electronics, vol. 20, no. 4, pp. 963-973, July 2005.

[3] Chia-Hung Lin et al., "Maximum photovoltaic power tracking for the PV array using the fractional-order incremental conductance method," Applied Energy, Vol. 88, Issue 12, 2011, pp. 4840-4847. 
[4] E. Koutroulis et al., "Development of a microcontroller-based, photovoltaic maximum power point tracking control system," IEEE Transactions on Power Electronics, vol. 16, no. 1, pp. 46-54, Jan. 2001.

[5] B. N. Alajmi et al., "Fuzzy-logic-control approach of a Modified Hill-Climbing Method for maximum power point in microgrid standalone photovoltaic system," IEEE Transactions on Power Electronics, vol. 26 , no. 4, pp. 1022-1030, Apr. 2011

[6] Syafaruddin et al., "Artificial neural network-polar coordinated fuzzy controller based maximum power point tracking control under partially shaded conditions," IET Renew. Power Gener., vol. 3, no. 2 , 239-253,2009.

[7] B. Lin, L. Wang and Q. Wu, "Maximum power point scanning for PV systems under various partial shading conditions," IEEE Transactions on Sustainable Energy, vol. 11, no. 4, pp. 2556-2566, Oct. 2020.

[8] M. Kermadi, Z. Salam, J. Ahmed and E. M. Berkouk, "A high-performance global maximum power point tracker of PV system for rapidly changing partial shading conditions," IEEE Transactions on Industrial Electronics, vol. 68, no. 3, pp. 2236-2245, March 2021.

[9] J. H. Holland, Adaptation in Natural and Artificial Systems: An Introductory Analysis With Applications to Biology, Control, and Artificial Intelligence. Cambridge, MA, USA: MIT Press, 1992.

[10] R. Storn and K. Price, "Differential Evolution - A simple and efficient heuristic for global optimization over continuous spaces," Journal of Global Optimization 11, 341-359 (1997).

[11] A. Messai et al., "Maximum power point tracking using a GA optimized fuzzy logic controller and its FPGA implementation," Solar Energy, vol.85,2011,265.

[12] K. S. Tey et al., "Improved differential evolution-based MPPT algorithm using SEPIC for PV systems under partial shading conditions and load variation," IEEE Transactions on Industrial Informatics, vol. 14, no. 10 , 4322-4333, 2018.

[13] M. Kermadi, Z. Salam, J. Ahmed and E. M. Berkouk, "An effective hybrid maximum power point tracker of photovoltaic arrays for complex partial shading conditions," IEEE Transactions on Industrial Electronics, vol. 66, no. 9, pp. 6990-7000, Sept. 2019.

[14] M. Seyedmahmoudian, T. K. Soon, B. Horan, A. Ghandhari, S. Mekhilef and A. Stojcevski, "New ARMO-based MPPT technique to minimize tracking time and fluctuation at output of PV systems under rapidly changing shading conditions," IEEE Transactions on Industrial Informatics, doi: 10.1109/TII.2019.2495066.

[15] N. Priyadarshi, S. Padmanaban, J. B. Holm-Nielsen, F. Blaabjerg and M. S. Bhaskar, "An experimental estimation of hybrid ANFIS-PSO-based MPPT for PV grid integration under fluctuating sun irradiance," IEEE Systems Journal, vol. 14, no. 1, pp. 1218-1229, March 2020

[16] N. Singh, K. K. Gupta, S. K. Jain, N. K. Dewangan and P. Bhatnagar, "A flying squirrel search optimization for MPPT under partial shaded photovoltaic system," IEEE Journal of Emerging and Selected Topics in Power Electronics, vol. 9, no. 4, pp. 4963-4978, Aug. 2021.

[17] D. S. Pillai, J. P. Ram, A. M. Y. M. Ghias, M. A. Mahmud and N. Rajasekar, "An accurate, shade detection-based hybrid maximum power point tracking approach for PV systems," IEEE Transactions on Power Electronics, vol. 35, no. 6, pp. 6594-6608, June 2020.

[18] Y. -P. Huang, M. -Y. Huang and C. -E. Ye, "A fusion firefly algorithm with simplified propagation for photovoltaic MPPT under partial shading conditions," IEEE Transactions on Sustainable Energy, vol. 11, no. 4, pp. 2641-2652, Oct. 2020

[19] S. Makhloufi and S. Mekhilef, "Logarithmic PSO based global/local maximum power point tracker for partially shaded photovoltaic systems," IEEE Journal of Emerging and Selected Topics in Power Electronics, doi 10.1109/JESTPE.2021.3073058.

[20] I. Shams, S. Mekhilef and K. S. Tey, "Maximum power point tracking using modified butterfly optimization algorithm for partial shading, uniform shading, and fast varying load conditions," IEEE Transactions on Power Electronics, vol. 36, no. 5, pp. 5569-5581, May 2021.

[21] H. Li et al., "An overall distribution particle swarm optimization MPPT algorithm for photovoltaic system under partial shading," IEEE Transactions on Industrial Electronics, vol. 66, no. 1, pp. 265-275, Jan. 2019.

[22] Duan, H. and Qiao, P. (2014), "Pigeon-inspired optimization: a new swarm intelligence optimizer for air robot path planning", International Journal of Intelligent Computing and Cybernetics, Vol. 7 No. 1, pp. 24-37.

[23] Daifeng Zhang and Haibin Duan, "Social-class pigeon-inspired optimization and time stamp segmentation for multi-UAV cooperative path planning," Neurocomputing, Vol. 313, 2018, pp. 229-246.

[24] IA, Z. (2016), "A type of collective detection scheme with improved pigeon-inspired optimization," International Journal of Intelligent Computing and Cybernetics, Vol. 9 No. 1, pp. 105-123.

[25] Kashif Ishaque et al., "Modeling and simulation of photovoltaic (PV) system during partial shading based on a two-diode model, Simulation Modelling Practice and Theory," Vol. 19, Issue 7, 2011, pp. 1613-1626.

[26] Tim Guilford et al., "Positional entropy during pigeon homing II: navigational interpretation of Bayesian latent state models," Journal of Theoretical Biology, Vol. 227, Issue 1, 2004, pp. 25-38.

[27] M. Nagy et al., "Hierarchical group dynamics in pigeon flocks," Nature, 464, 890-893 (2010).

[28] M. Nagy et al., "Context-dependent hierarchies in pigeons," Proceedings of the National Academy of Sciences, Aug 2013, 110 (32) 13049-13054.

[29] K. Ishaque and Z. Salam, "A deterministic particle swarm optimization maximum power point tracker for photovoltaic system under partial shading condition," IEEE Transactions on Industrial Electronics, vol. 60, no. 8, pp. 3195-3206, Aug. 2013.

[30] D. F. Teshome, C. H. Lee, Y. W. Lin and K. L. Lian, "A modified firefly algorithm for photovoltaic maximum power point tracking control under partial shading," IEEE Journal of Emerging and Selected Topics in Power Electronics, vol. 5, no. 2, pp. 661-671, June 2017.

[31] C. Huang, L. Wang, R. S. Yeung, Z. Zhang, H. S. Chung and A. Bensoussan, "A prediction model-guided Jaya algorithm for the PV system maximum power point tracking," IEEE Transactions on Sustainable Energy, vol. 9, no. 1, pp. 45-55, Jan. 2018.

[32] M. A. Ghasemi, H. M. Foroushani and M. Parniani, "Partial Shading Detection and Smooth Maximum Power Point Tracking of PV Arrays Under PSC," IEEE Transactions on Power Electronics, vol. 31, no. 9, pp. 6281-6292, Sept. 2016.

[33] A. Gholami, M. Ameri, M. Zandi, R. G. Ghoachani, S. Eslami and S. Pierfederici, "Photovoltaic potential assessment and dust impacts on photovoltaic systems in Iran: Review paper," IEEE Journal of Photovoltaics, vol. 10, no. 3, pp. 824-837, May 2020

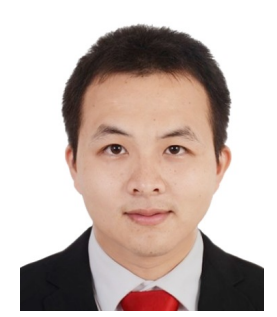

Zhuoli Zhao (S'15-M'18) received the Ph.D. degree in electrical engineering from South China University of Technology, Guangzhou, China, in 2017. From October 2014 to December 2015, he was a Joint Ph.D. Student (Sponsored Researcher) with the Control and Power Research Group, Department of Electrical and Electronic Engineering, Imperial College London, London, U.K. He was a Research Associate with the Smart Grid Research Laboratory, Electric Power Research Institute, China Southern Power Grid, Guangzhou, China, from 2017 to 2018.

$\mathrm{He}$ is currently an Associate Professor with the School of Automation, Guangdong University of Technology, Guangzhou, China. His research interests include microgrid control and energy management, power electronic converters, smart grids, and distributed generation systems. $\mathrm{He}$ is an Active Reviewer for the IEEE Transactions on Smart Grid, the IEEE Transactions on Power Electronics, the IEEE Transactions on Sustainable Energy, the IEEE Transactions on Industrial Electronics, and the Applied Energy.

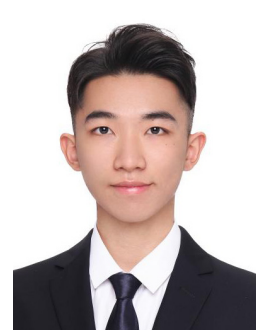

Mingyu Zhang is currently working toward the bachelor's degree in electrical engineering with the School of Automation, Guangdong University of Technology, Guangzhou, China. His research interests include power electronic converters and smart grids. 


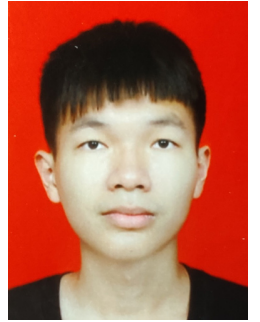

Zehan Zhang is currently working toward the bachelor's degree in electrical engineering with the School of Automation, Guangdong University of Technology, Guangzhou, China. His research interests include embedded control and energy management in power electronic converters and smart grids.

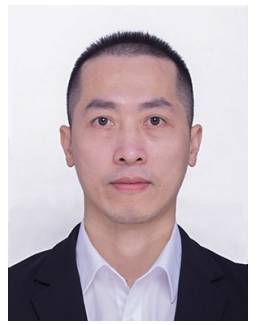

Yuewu Wang received his B.S. degree in Instrument Science and Technology from Xi'an Jiaotong University, Xi'an, China, in 2006, his M.S. degree in Electrical Engineering from Guangxi University, Nanning, China, in 2010 and his Ph.D. degree in Electrical Engineering from South China University of Technology, Guangzhou, China, in 2017.

From 2017 to 2020, he was a Software Engineer with Shenzhen INVT Electric Co., Ltd. Shenzhen, China. In 2020, he joined the School of Electric and Information Engineering, Guangxi University of Science and Technology, Liuzhou, China, where he is currently a Lecturer. His current research interests include pulse width modulation techniques, DC-DC converters and grid-connected inverters.

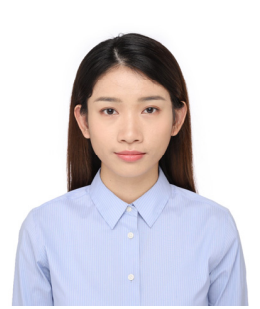

systems.
Runting Cheng received the B.Eng. degree in electrical engineering and automation from Northeast Forestry University, Harbin, China, in 2018, and the M.S. degree in Electrical Engineering from Guangdong University of Technology, Guangzhou, China, in 2021. She is currently a research assistant with South China University of Technology, Guangzhou, China. Her research interests include distribution network dispatching and distributed generation

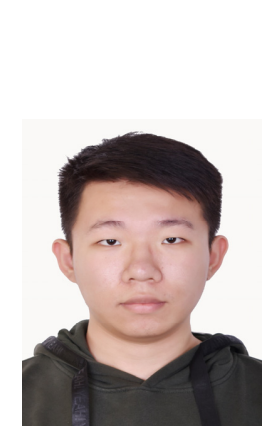

Juntao Guo received the B.E. degree from Guangdong University of Technology, Guangzhou, China, in 2019. He is currently working toward the master degree in electrical engineering with the School of Automation, Guangdong University of Technology, Guangzhou, China. His research interests include microgrid control and energy management.

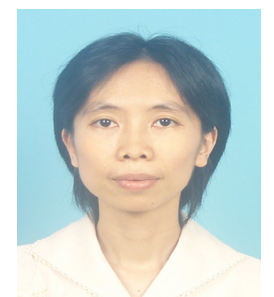

Ping Yang (M'11) received the Ph.D. degree in automatic control from South China University of Technology, Guangzhou, China, in 1998. She is currently a Professor with the School of Electric Power Engineering, South China University of Technology, Guangzhou, China and the Director of Guangdong Key Laboratory of Clean Energy Technology, South China University of Technology, Guangzhou, China. Her current research interests include smart microgrid and

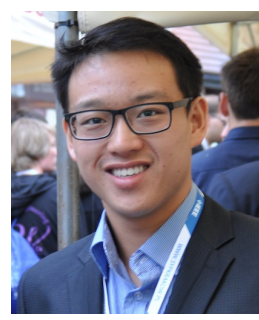

Chun Sing Lai (S'11, M'19, SM'20) received the B.Eng. (First Class Hons.) in electrical and electronic engineering from Brunel University London, London, UK, in 2013, and the D.Phil. degree in engineering science from the University of Oxford, Oxford, UK, in 2019.

$\mathrm{He}$ is currently a Lecturer with the Department of Electronic and Electrical Engineering, Brunel University London. From 2018 to 2020, he was an UK Engineering and Physical Sciences Research Council Research Fellow with the School of Civil Engineering, University of Leeds, Leeds, UK. His current research interests are in power system optimization and data analytics.

Dr. Lai was the Publications Co-Chair for both 2020 and 2021 IEEE International Smart Cities Conferences. He is the Vice-Chair of the IEEE Smart Cities Publications Committee and Associate Editor for IET Energy Conversion and Economics. He is the Working Group Chair for IEEE P2814 Standard, and the Chair of the IEEE SMC Intelligent Power and Energy Systems Technical Committee. He is an IET Member and a Chartered Engineer.

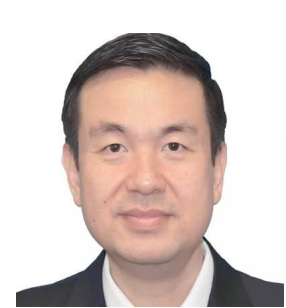

Peng Li received the B.Sc., M.Sc., and Ph.D. degrees from South China University of Technology, Guangzhou, China, in 1993, 1995, and 2002, respectively, and the Ph.D. degree from the Technical University of Braunschweig, Braunschweig, Germany, in 2004, all in electrical engineering. $\mathrm{He}$ is currently a Professor-level Senior Engineer of the Digital Grid Research Institute of China Southern Power Grid, Guangzhou, China. His current research interests include technology research and management of smart grid and digital grid.

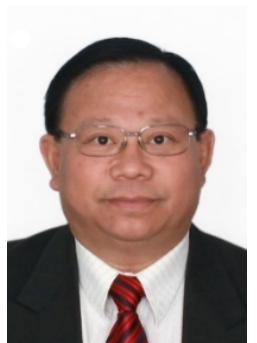

Loi Lei Lai (M'87, SM'92, F'07, LF'21) received the B.Sc. (First Class Hons.), Ph.D., and D.Sc. degrees in electrical and electronic engineering from the University of Aston, Birmingham, UK, and City, University of London, London, UK, in 1980, 1984, and 2005, respectively.

Professor Lai is currently a University Distinguished Professor with Guangdong University of Technology, Guangzhou, China. He was a Pao Yue Kong Chair Professor with Zhejiang University, Hangzhou, China, and the Professor and Chair of Electrical Engineering with City, University of London. His current research areas are in smart cities and smart grid. Professor Lai was awarded an IEEE Third Millennium Medal, the IEEE Power and Energy Society (IEEE/PES) UKRI Power Chapter Outstanding Engineer Award in 2000, the IEEE/PES Energy Development and Power Generation Committee Prize Paper in 2006 and 2009, the IEEE/SMCS Outstanding Contribution Award in 2013 and 2014, the Most Active Technical Committee Award in 2016, and his research team has received a Best Paper Award in the IEEE International Smart Cities Conference in October 2020.

Professor Lai is an Associate Editor of the IEEE Transactions on Systems, Man, and Cybernetics: Systems, Editor-in-Chief of the IEEE Smart Cities Newsletter, a member of the IEEE Smart Cities Steering Committee and the Chair of the IEEE Systems, Man, and Cybernetics Society (IEEE/SMCS) Standards Committee. He was a member of the IEEE Smart Grid Steering Committee; the Director of Research and Development Center, State Grid Energy Research Institute, China; a Vice President for Membership and Student Activities with IEEE/SMCS; and a Fellow Committee Evaluator for the IEEE Industrial Electronics Society. He is a Fellow of IET. 